\title{
Two nanoformulations induce reactive oxygen species and immunogenetic cell death for synergistic chemo- immunotherapy eradicating colorectal cancer and hepatocellular carcinoma
}

\author{
Jianfeng Guo ${ }^{1,2}$, Zhuo Yu ${ }^{2,3}$, Dandan Sun ${ }^{1}$, Yifang Zou' ${ }^{1}$ Y Yun Liu² and Leaf Huang ${ }^{2 *}$
}

\begin{abstract}
Background: FOLFOX is a combinational regimen of folinic acid (FnA, FOL), fluorouracil (5-Fu, F) and oxaliplatin (OxP, OX), and has been long considered as the standard treatment of colorectal cancer (CRC) and hepatocellular carcinoma (HCC). Recent developments of nano delivery systems have provided profound promise for improving anticancer efficacy and alleviating side effects of FOLFOX. Previously, a nanoformulation (termed Nano-Folox) containing OxP derivative and FnA was developed in our laboratory using nanoprecipitation technique. Nano-Folox induced OxP-mediated immunogenic cell death (ICD)-associated antitumor immunity, which significantly suppressed tumor growth in the orthotopic CRC mouse model when administrated in combination with free 5-Fu.

Methods: A nanoformulation (termed Nano-FdUMP) containing FdUMP (5-Fu active metabolite) was newly developed using nanoprecipitation technique and used in combination with Nano-Folox for CRC and HCC therapies.

Results: Synergistic efficacy was achieved in orthotopic CRC and HCC mouse models. It resulted mainly from the fact that Nano-FdUMP mediated the formation of reactive oxygen species (ROS), which promoted the efficacy of ICD elicited by Nano-Folox. In addition, combination of Nano-Folox/Nano-FdUMP and anti-PD-L1 antibody significantly inhibited CRC liver metastasis, leading to long-term survival in mice.

Conclusion: This study provides proof of concept that combination of two nano delivery systems can result in successful FOLFOX-associated CRC and HCC therapies. Further optimization in terms of dosing and timing will enhance clinical potential of this combination strategy for patients.
\end{abstract}

Keywords: Nanoparticles, Drug delivery, Immunogenic cell death, Reactive oxygen species, Combination therapy

\footnotetext{
* Correspondence: leafh@email.unc.edu

${ }^{2}$ Division of Pharmacoengineering and Molecular Pharmaceutics, Eshelman School of Pharmacy, University of North Carolina, Chapel Hill, NC 27599, USA

Full list of author information is available at the end of the article
}

(c) The Author(s). 2021 Open Access This article is licensed under a Creative Commons Attribution 4.0 International License, which permits use, sharing, adaptation, distribution and reproduction in any medium or format, as long as you give appropriate credit to the original author(s) and the source, provide a link to the Creative Commons licence, and indicate if changes were made. The images or other third party material in this article are included in the article's Creative Commons licence, unless indicated otherwise in a credit line to the material. If material is not included in the article's Creative Commons licence and your intended use is not permitted by statutory regulation or exceeds the permitted use, you will need to obtain permission directly from the copyright holder. To view a copy of this licence, visit http://creativecommons.org/licenses/by/4.0/ The Creative Commons Public Domain Dedication waiver (http://creativecommons.org/publicdomain/zero/1.0/) applies to the data made available in this article, unless otherwise stated in a credit line to the data. 


\section{Introduction}

The FOLFOX regimen including folinic acid (FnA), fluorouracil $(5-\mathrm{Fu})$ and oxaliplatin $(\mathrm{OxP})$ has been used as the standard chemotherapy for patients with colorectal cancer (CRC) and hepatocellular carcinoma (HCC) at advanced stages $[1,2]$. FOLFOX has also provided therapeutic benefits for patients with unresectable CRC liver metastases [3]. The clinical practice of FOLFOX includes: 1) patients are intravenously (i.v.) infused with OxP and FnA simultaneously, which are followed by i.v. administration of 5 -Fu; 2) patients receive FOLFOX for 8 to 12 cycles every 2 to 3 weeks. Once inside tumor cells, the activity of $5-\mathrm{Fu}$ is enhanced by $\mathrm{FnA}$, and $5-\mathrm{Fu} /$ FnA adds or synergizes with OxP $[1,2]$. Notably, major barriers associated with FOLFOX including non-specific delivery, high toxicity and long course of treatment still limits its clinical application [4,5]. It is widely established that nanomaterials may be designed for tissueand cell-specific delivery of chemotherapeutics, which will improve therapeutic efficacy against tumors and reduce damage to healthy tissues [6]. Therefore, development of nano delivery systems holds great promise for overcoming the barriers associated with FOLFOX.

We have recently produced a nanoscale precipitate $\left(\mathrm{C}_{26} \mathrm{H}_{35} \mathrm{~N}_{9} \mathrm{O}_{7} \mathrm{Pt}\right)$ using the adduct of [Pt $\left.(\mathrm{DACH})\left(\mathrm{H}_{2} \mathrm{O}\right)_{2}\right]^{2+}$ (active form of OxP) and $\mathrm{FnA}^{2-}$ [7]. $\mathrm{C}_{26} \mathrm{H}_{35} \mathrm{~N}_{9} \mathrm{O}_{7} \mathrm{Pt}$ could be encapsulated into an aminoethyl anisamide (AEAA)-targeted polyethylene glycol (PEG)modified lipid nanoparticle (NP) using nanoprecipitation process, forming a nanoformulation termed Nano-Folox [7]. AEAA is a high affinity $(\mathrm{Kd} \approx 9 \mathrm{nM})$ ligand for the sigma-1 receptor which is overexpressed in most of solid tumor cells [8-11]. Nano-Folox induced robust anticancer immunity, which is mainly due to OxP-mediated immunogenic cell death (ICD) [12]. When administrated in combination with free 5-Fu, Nano-Folox at lower doses led to significantly enhanced tumor regression in an orthotopic CRC mouse model than FOLFOX, i.e., free drugs in combination, at higher doses. Moreover, a combination strategy "Nano-Folox/5-Fu and anti-PD-L1 monoclonal antibody (mAb)" remarkably slowed down CRC liver metastasis. It is worth noting that although tumor growth was significantly inhibited during treatment period, tumors progressed rapidly after dosing, and no long-term survival was achieved in mice. These results imply that tumor recurrence is caused most likely due to the lack of tumor-specific memory immune response.

It has been reported that the differentiation of naive $T$ cells is highly associated with the antigen availability to DCs [13], and higher amount and longer duration of antigen stimulation produce larger number of effector and memory $\mathrm{T}$ cells [14]. ICD can induce the exposure of damage-associated molecular patterns (DAMPs) from dying or dead cancer cells, resulting in the antigen presentation to DCs for tumor-specific $\mathrm{T}$ cell response [15]. It has also been reported that the induction of ICD is accompanied with the formation of reactive oxygen species (ROS) [15], and the ICD efficacy may be enhanced by ROS-inducing strategies [16-18]. Therefore, we hypothesize that the ROS induction may be safely and effectively achieved by targeted delivery of 5 -Fu using nano delivery systems, which will synergize with Nano-Folox to induce effector and memory $T$ cells for tumor-specific killing and protective responses.

Consequently, an AEAA-targeted PEGylated lipid NP (termed Nano-FdUMP) was newly produced using nanoprecipitation technique for delivery of 5-Fluoro-2' -deoxyuridine $5^{\prime}$-monophosphate (FdUMP, an active 5-Fu metabolite) [19]. The chemo-immunotherapeutic efficacy of Nano-FdUMP was investigated using orthotopic and metastatic mouse models when applied alone or in combination with Nano-Folox and anti-PD-L1 mAb.

\section{Methods \\ Materials}

5-Fluoro-2'-deoxyuridine 5'-monophosphate (FdUMP), 2 '-deoxyuridine 5 '-monophosphate (dUMP), dichloro (1, 2-diaminocyclohexane) platinum (II), $\mathrm{AgNO}_{3}, \mathrm{IGEPAL}^{\circ}$ CO-520, cyclohexane, Triton X-100, $\mathrm{CaCl}_{2},\left(\mathrm{NH}_{4}\right)_{2} \mathrm{HPO}_{4}$, cholesterol, folinic acid ( $\mathrm{FnA})$ and 5-Fluorouracil (5-Fu) were obtained from Sigma-Aldrich. Oxaliplatin (OxP) was obtained from Selleckchem. 1,2-dioleoyl-sn-glycero-3phosphate (DOPA) and 1,2-dioleoyl-3-trimethylammonium-propane (DOTAP) were purchased from Avanti Polar Lipids. N-(Carbonyl-methoxypolyethyleneglycol 2000)-1,2-distearoyl-sn-glycero-3-phosphoethanolamine

(SUNBRIGHT ${ }^{\otimes}$ DSPE-020CN; DSPE-PEG) was obtained from NOF CORP. DSPE-PEG-AEAA was synthesized as previously demonstrated by our laboratory [20].

\section{Preparation and characterization of nanoformulations}

Nano-FdUMP was prepared as previously described with modifications [21, 22]. Briefly, $1 \mathrm{~mL}$ of FdUMP solution $(1 \mathrm{mg} / \mathrm{mL})$ was added into $2 \mathrm{~mL}$ of $\mathrm{CaCl}_{2}$ solution $(2.5$ $\mathrm{M})$, and this mixture was added into $80 \mathrm{~mL}$ oil phase composed of IGEPAL ${ }^{\circ}$ CO-520 and cyclohexane (30:70, $\mathrm{V}: \mathrm{V})$ for the generation of water-in-oil reverse microemulsion. Another microemulsion $(80 \mathrm{~mL})$ was prepared by adding $2 \mathrm{~mL}$ of $\left(\mathrm{NH}_{4}\right)_{2} \mathrm{HPO}_{4}$ solution $(50 \mathrm{mM})$ and 1 $\mathrm{mL}$ of DOPA solution ( $20 \mathrm{mM}$ in chloroform). Two microemulsions were stirred for $\sim 15$ to $20 \mathrm{~min}$. After this, $160 \mathrm{~mL}$ of ethanol were added for $\sim 15$ to $20 \mathrm{~min}$ with stirring, which was followed by centrifugation for $20 \mathrm{~min}$ at $10,000 \mathrm{~g}$ for collection of nanoprecipitates. Nanoprecipitates were washed using ethanol, dried using nitrogen, and stored in chloroform.

The optimal ratio between nanoprecipitates and outer leaflet lipids for Nano-FdUMP was as follows: $1500 \mu \mathrm{g}$ of 
nanoprecipitates, $30 \mu \mathrm{L}$ of DOTAP $(25 \mathrm{mM}), 30 \mu \mathrm{L}$ cholesterol $(25 \mathrm{mM})$ and $20 \mu \mathrm{L}$ DSPE-PEG/DSPE-PEGAEAA $(20 \mathrm{mM}$, molar ratio $=5: 1)$ in $2 \mathrm{~mL}$ of chloroform. This theoretically achieved $\sim 3.5 \mathrm{~mol} \%$ of AEAA on the outer lipid surface per formulation. Following the evaporation of chloroform, the lipid film was resuspended to form Nano-FdUMP aqueous solution. The encapsulation efficiency and loading capacity were assessed using HPLC (Shimadzu, Japan) (C18 column, UV at $250 \mathrm{~nm}$, mobile phase $=$ water and methanol, 85:15). Nano-dUMP and non-targeted Nano-FdUMP were prepared as mentioned above except the use of dUMP and the lack of DSPE-PEG-AEAA, respectively.

In addition, Nano-Folox was prepared as previously described [7]. Briefly, $\mathrm{AgNO}_{3}(64.5 \mathrm{mg}, 0.38 \mathrm{mmol})$ was added with dichloro (1,2-diaminocyclohexane) platinum (II) $(76 \mathrm{mg}, 0.2 \mathrm{mmol}$ ) in $1 \mathrm{~mL}$ deionized water, in order to produce the dihydrate (1,2-diaminocyclohexane) platinum (II). After this, the solution was heated at $\sim 60^{\circ} \mathrm{C}$ for $3 \mathrm{~h}$ and continued with stirring at RT overnight. Subsequently, the concentration of dihydrate (1,2-diaminocyclohexane) platinum (II) was measured using inductively coupled plasma mass spectrometry (ICP-MS) [23]. $100 \mu \mathrm{L}$ dihydrate (1,2-diaminocyclohexane) platinum (II) (100 $\mathrm{mM}$ ) were added into a $25 \mathrm{~mL}$ oil phase (hexanol, Triton $\mathrm{X}-100$ and cyclohexane; $10: 15: 75, \mathrm{~V}: \mathrm{V}: \mathrm{V})$ for the production of water-in-oil reverse microemulsion. Moreover, 2 $\mathrm{mL}$ FnA $(10 \mathrm{mM})$ and $200 \mu \mathrm{L}$ DOPA $(20 \mathrm{mM})$ were added into a $75 \mathrm{~mL}$ oil phase with stirring to produce another water-in-oil reverse microemulsion. After $15 \mathrm{~min}$, two microemulsions were mixed for $\sim 45 \mathrm{~min}$ with stirring. Subsequently, $100 \mathrm{~mL}$ ethanol were added with stirring for $\sim 15 \mathrm{~min}$, which were followed by the centrifugation for $\sim 20 \mathrm{~min}$ at $10,000 \mathrm{~g}$ to collect nanoprecipitates $\left(\mathrm{C}_{26} \mathrm{H}_{35} \mathrm{~N}_{9} \mathrm{O}_{7} \mathrm{Pt}\right)$. For $1 \mathrm{mg}$ nanoprecipitate core, $10 \mu \mathrm{L}$ of $20 \mathrm{mM}$ DOTAP, $10 \mu \mathrm{L}$ of $20 \mathrm{mM}$ cholesterol and $5 \mu \mathrm{L} 20$ $\mathrm{mM}$ DSPE-PEG/DSPE-PEG-AEAA (molar ratio $=4: 1$ ) were added into $1 \mathrm{~mL}$ chloroform. After evaporating chloroform, the lipid film was reconstituted using deionized water to form Nano-Folox.

The hydrodynamic diameter and zeta potential of NPs were measured using Malvern Nano-ZS. The morphology of NPs was observed using the JEM1230 (JEOL) transmission electron microscope (TEM). In addition, a solution of NPs with $200 \mu \mathrm{g}$ of FdUMP was incubated at $37^{\circ} \mathrm{C}$ in $0.01 \mathrm{M}$ PBS ( $\mathrm{pH}=5.5$ and 7.4 ) with shaking. Samples were obtained at different time points for centrifugation at $10,000 \mathrm{~g}$ for $\sim 30 \mathrm{~min}$. The concentration of free FdUMP within supernatants (dissociated from nanoprecipitates) was determined using HPLC.

\section{Cell culture}

CT26 (mouse CRC cell line), Hepa1-6 (mouse HCC cell line), 4 T1 (mouse breast cancer cell line) and B16 (mouse melanoma cell line) cells were cultured using DMEM (Gibco) with 10\% bovine calf serum (Hyclone) and $1 \%$ antibiotic-antimycotic (Gibco). CT26-FL3 (a subtype of CT26, it is engineered to stably express luciferase) and Hepa1-6-Luc (it is engineered to stably express luciferase) cells $[7,24]$ were cultured using the aforementioned growth medium with $1 \mu \mathrm{g} / \mathrm{mL}$ puromycin (ThermoFisher). Cells were maintained at $37^{\circ} \mathrm{C}$ with $5 \% \mathrm{CO}_{2}$ and $95 \%$ relative humidity.

\section{In vitro studies}

MTT assay was applied to determine in vitro cytotoxicity. CT26 and Hepa1-6 cells $\left(1 \times 10^{4} /\right.$ well $)$ were cultured within 96-well plates, respectively. Following one day incubation, 5-Fu, Nano-dUMP and Nano-FdUMP were added to cells for $24 \mathrm{~h}$. Cells were then added with MTT reagent at $37^{\circ} \mathrm{C}$ for $\sim 4 \mathrm{~h}$ before measurement at $570 \mathrm{~nm} . \mathrm{IC}_{50}$ was calculated using the GraphPad Prism software.

CT26 and Hepa1-6 cells $\left(5 \times 10^{4} /\right.$ well $)$ were placed into 24-well plates, respectively. After one day incubation, cells were treated with or without $\mathrm{N}$-acetylcysteine (NAC; $5 \mathrm{mM}$ ) for $4 \mathrm{~h}$. Cells were replaced with fresh growth medium and added with 5-Fu, Nano-dUMP and Nano-FdUMP (all at $15 \mu \mathrm{M}$ ) for $24 \mathrm{~h}$. Subsequently, apoptotic cells were detected using Annexin V-FITC/ propidium iodide assay (Promega) and measured by the Becton Dickinson FACSCalibur. In a separate experiment, the ROS level in cells was detected using $2^{\prime}, 7^{\prime}$ dichlorodihydrofluorescein diacetate-based Reactive Oxygen Species Assay Kit (YIASEN Biotech) by microplate reader $(488 \mathrm{~nm} / 525 \mathrm{~nm})$.

The CRT exposure was detected using immunofluorescence staining assay. CT26 and Hepa1-6 cells (60,000 per well) were cultured in 8-well chamber slides (ThermoFisher). Following one day incubation, cells were treated with or without NAC $(5 \mathrm{mM})$ for $4 \mathrm{~h}$. Cells were then replaced with fresh growth medium and treated with either Nano-FdUMP $(15 \mu \mathrm{M})$, Nano-Folox $(5 \mu \mathrm{M})$, or both (Nano-Folox was first added, and FdUMP was added at $2 \mathrm{~h}$ later; this sequential administration was same for in vitro studies unless mentioned otherwise). Two $h$ post treatment, cells were incubated with $0.25 \%$ paraformaldehyde (PFA). Following $5 \mathrm{~min}$ incubation, cells were washed with PBS, which were followed by the application of anti-CRT antibody (ab2907, Abcam, 1: $500)$ for $1 \mathrm{~h}$. After PBS washes, FITC-conjugated secondary antibodies (ab150077, Abcam) were added into cells for $30 \mathrm{~min}$. Subsequently, cells were treated by $4 \%$ PFA for $20 \mathrm{~min}$ and stained using DAPI (ThermoFisher) for confocal imaging (LSM-710, Zeiss).

In order to measure the extracellular ATP, CT26 and Hepa1-6 cells were placed into 24-well plates at a density of 60,000 cells per well. After one day incubation, 
cells were treated with or without NAC ( $5 \mathrm{mM})$ for $4 \mathrm{~h}$. Cells were replaced with fresh growth medium and added with either Nano-FdUMP $(15 \mu \mathrm{M})$, Nano-Folox $(5 \mu \mathrm{M})$, or both for $24 \mathrm{~h}$. Subsequently, extracellular ATP was detected using ENLITEN ${ }^{\circ}$ ATP Assay System Bioluminescence Detection Kit.

The release of HMGB1 was analyzed using ELISA assay. CT26 and Hepa1-6 cells (60,000 per well) were cultured in 24-well plates. Following one day incubation, cells were treated with or without NAC $(5 \mathrm{mM})$ for $4 \mathrm{~h}$. Cells were replaced with fresh growth medium and added with either Nano-FdUMP $(15 \mu \mathrm{M})$, Nano-Folox $(5 \mu \mathrm{M})$, or both for $8 \mathrm{~h}$. After this, the level of HMGB1 in the supernatants was measured using ELISA kit (LSF11641, LifeSpan BioSciences).

\section{In vivo toxicity, pharmacokinetics and biodistribution}

Six-week old female BALB/C and male C57BL/6 mice were purchased from Charles River Laboratories. The procedures used in this study were approved by Institutional Animal Care and Use Committee of University of North Carolina at Chapel Hill and by the Animal Ethics Committee of Jilin University.

Healthy mice were treated with nanoformulations as described in Figs. S2 and S9 $(n=5)$. Body weight was recorded, and the whole blood and the serum of animals were obtained on Day 35 to analyze myelosuppression and hepatic/renal functions.

The orthotopic CRC mouse model was achieved as previously described [7]. Briefly, BALB/C mice were anesthetized by $2.5 \%$ isoflurane, and the cecum wall was injected with $\sim 1 \times 10^{6}$ CT26-FL3 cells. In addition, the orthotopic HCC mice were established as previously described [25]. Briefly, C57BL/ 6 mice were anesthetized by $2.5 \%$ isoflurane, and the liver was injected with $\sim 1 \times 10^{6}$ Hepa1-6Luc cells. Following tumor inoculation (Day 0), animals were intraperitoneally (i.p.) injected with $100 \mu \mathrm{L}$ luciferin $\left(10 \mathrm{mg} / \mathrm{mL}\right.$; Pierce $\left.{ }^{\mathrm{rm}}\right)$, and tumor growth was measured using IVIS ${ }^{\bullet}$ Kinetics Optical System (Perkin Elmer). When tumor growth was reached at $\sim 0.5$ to $1 \times 10^{9} \mathrm{p} / \mathrm{sec} / \mathrm{cm}^{2} /$ sr, pharmacokinetics and tissue distribution studies were investigated as follows: 1) 5 - Fu $(10 \mathrm{mg} / \mathrm{kg})$ or NanoFdUMP containing $10 \mathrm{mg} / \mathrm{kg}$ of fluorine drug were i.v. administrated, and the blood $(\sim 50 \mu \mathrm{L})$ was collected at 1,5 , 10 , and $15 \mathrm{~min}$, and $0.5,1,4,8$ and $12 \mathrm{~h}(n=4)$. As previously described [26], plasma samples were extracted with ethyl acetate, dried with nitrogen, and reconstituted in the mobile phase (water/methanol, 85:15). The concentration was assessed using HPLC (Shimadzu, Japan) (C18 column, $\mathrm{UV}$ at $265 \mathrm{~nm}$ for 5 -Fu and UV at $250 \mathrm{~nm}$ for FdUMP). Half-life was evaluated using DAS 2.0 software. In separate studies, $\sim 0.05 \mathrm{wt} \%$ of $\mathrm{DiD}$ (ThermoFisher) was formulated into Nano-FdUMP or non-targeted counterpart (10 $\mathrm{mg} / \mathrm{kg}$ of fluorine drug). Twelve h post i.v. administration, distribution of DiD-labeled nanoformulations into tissues and tumors was detected $(640 \mathrm{~nm} / 670 \mathrm{~nm})$ using IVIS ${ }^{\circ}$ Kinetics Optical System $(n=4)$.

\section{Synergistic efficacy of nano-FdUMP and nano-Folox in orthotopic CRC and HCC mouse models}

When tumor growth was reached at $\sim 0.5$ to $1 \times 10^{9} \mathrm{p} / \mathrm{sec} /$ $\mathrm{cm}^{2} / \mathrm{sr}$, tumor-bearing mice were injected with either $\mathrm{OxP} /$ FnA $(1.5 \mathrm{mg} / \mathrm{kg}$ and $4.5 \mathrm{mg} / \mathrm{kg}$, i.v.) or Nano-Folox containing $1.5 \mathrm{mg} / \mathrm{kg}$ of platinum drug (i.v.; due to the molar ratio of OxP derivative and $\mathrm{FnA}=1: 1$ for nanoprecipitate $\left(\mathrm{C}_{26} \mathrm{H}_{35} \mathrm{~N}_{9} \mathrm{O}_{7} \mathrm{Pt}\right)$ [7], it contained $\sim 4.5 \mathrm{mg} / \mathrm{kg}$ of $\left.\mathrm{FnA}\right)$ as described in figs. 5 and 6 . Eight $h$ post injection $\left(t_{1 / 2}\right.$ of Nano-Folox $\approx 1.4 \mathrm{~h}$ ), tumor-bearing mice were treated with either 5 -Fu (10 mg/kg; i.v.) or Nano-FdUMP containing 10 $\mathrm{mg} / \mathrm{kg}$ of fluorine drug (i.v.). Tumor growth was observed using the IVIS ${ }^{\circ}$ Kinetics Optical System $(n=6)$.

In separate experiments, 3 days after two injections (time point to analyze chemotherapeutic and immunotherapeutic effects was generally chosen within one week following treatment to ensure reliable analyses) [10, 2729], tumors were obtained on Day 24 (CRC) and Day 23 (HCC) for following assays: 1) TUNEL assay [7, 24]. It was performed using the DeadEnd ${ }^{\mathrm{Tm}}$ Fluorometric TUNEL System (Promega) $(n=4)$. DNA fragments (FITC) and nuclei (DAPI) were detected by confocal microscopy; 2) Flow cytometry [7, 24]. Tumors $(n=4)$ were treated using collagenase A $(1 \mathrm{mg} / \mathrm{mL}$; Sigma) and DNAse $(200 \mu \mathrm{g} / \mathrm{mL}$; Invitrogen) for $30 \mathrm{~min}$ at $37^{\circ} \mathrm{C}$ to produce single cells. After lysis of erythrocytes with ACK buffer (Gibco), cells were treated by fluorophore-labeled antibodies (see Supplementary Table 1), fixed using 4\% PFA, and assessed using the Becton Dickinson LSR II. 3) RT-PCR assay [7, 24]. Total RNA samples $(n=4)$ were obtained using the Qiagen RNeasy ${ }^{\circ}$ Microarray Tissue Mini Kit. cDNA was generated by a BIO-RAD iScript ${ }^{\mathrm{tm}}$ cDNA Synthesis Kit. The RT-PCR reaction was carried out using the TaqMan Gene Expression Master Mix (BIO-RAD) by the 7500 Real-Time PCR System. The information of primers was shown in Supplementary Table 2.

The depletion study of $\mathrm{CD}_{4}^{+}$and $\mathrm{CD} 8^{+} \mathrm{T}$ cells was performed as previously described [7, 24]. In brief, $100 \mu \mathrm{g}$ of either anti-CD8 (clone 53-6.72, Bioxcell), antiCD4 (clone GK1.5, Bioxcell) or IgG (Bioxcell, polyclonal) antibodies were i.p. injected per mouse at respective schedules (figs. 5 and 6) before the treatment of NanoFdUMP/Nano-Folox. Tumor growth was measured using the IVIS ${ }^{\bullet}$ Kinetics Optical System $(n=4)$.

\section{Combination therapy of nano-FdUMP and nano-Folox with PD-L1 blockade for CRC liver metastasis mouse model}

The CRC liver metastasis mouse model was established as previously described [7]. In brief, mice were 
anesthetized using 2.5\% isoflurane, and the spleen was exteriorized, tied and sectioned. Afterwards, $\sim 2 \times 10^{5}$ CT26-FL3 cells were injected to the distal section of the spleen. The hemi-spleen injected by CT26-FL3 cells was removed, and the other half was placed back into the cavity. Following tumor inoculation at Day 0, tumor growth was monitored using the IVIS ${ }^{\circ}$ Kinetics Optical System. When tumor growth was reached at $\sim 0.5$ to $1 \times 10^{8} \mathrm{p} / \mathrm{sec} / \mathrm{cm}^{2} / \mathrm{sr}$, mice were i.v. administrated with Nano-Folox containing $1.5 \mathrm{mg} / \mathrm{kg}$ of Pt $(\sim 4.5 \mathrm{mg} / \mathrm{kg}$ of FnA) as described in fig. 7 , which were followed by i.v. administration of Nano-FdUMP $(10 \mathrm{mg} / \mathrm{kg}$ of fluorine drug) at $8 \mathrm{~h}$ post-injection. After this, mice were i.p. injected with or without anti-PD-L1 mAb (Bioxcell, clone 10F.9G2, $100 \mu \mathrm{g}$ per mouse). The tumor growth was observed using the IVIS ${ }^{\circ}$ Kinetics Optical System $(n=6)$. Separately, one day following two injections, tumors were obtained on Day 12 for TUNEL analysis $(n=$ $4)$, flow cytometry $(\mathrm{n}=4)$ and RT-PCR experiment $(\mathrm{n}=$ 4), as described above.

\section{Statistical analysis}

Data were presented in this work as mean \pm standard deviation (SD). The significance between two groups was evaluated using unpaired Student's t-test (two-tailed). The significance between three or more groups was assessed using the two-way ANOVA (Bonferroni's Post-
Hoc model). A log rank test was utilized for comparison in survival study. In this work, $p<0.05$ was considered statistically significant.

\section{Results}

\section{Preparation and physicochemical characterization of nano-FdUMP}

One water-in-oil microemulsion containing $\mathrm{CaCl}_{2}$ and FdUMP was mixed with another water-in-oil microemulsion containing $\mathrm{Na}_{2} \mathrm{HPO}_{4}$, in order to generate $\mathrm{Ca}_{3}\left(\mathrm{PO}_{4}\right)_{2}$ amorphous precipitate in which FdUMP was entrapped (fig. 1a). The $\mathrm{Ca}_{3}\left(\mathrm{PO}_{4}\right)_{2}$-FdUMP nanoprecipitate was stabilized by 1,2-dioleoyl-sn-glycero-3-phosphate (DOPA), and the stabilized nanoprecipitate was coated with 1,2-dioleoyl-3-trimethylammonium-propane (DOTAP), cholesterol, 1,2-distearoyl-sn-glycero-3-phosphoethanolamine-PEG ${ }_{2000}$ (DSPE-PEG) and DSPE-PEGAEAA, resulting in Nano-FdUMP (fig. 1b). NanoFdUMP is reminiscent of other nanoformulations containing $\mathrm{Ca}_{3}\left(\mathrm{PO}_{4}\right)_{2}$-nucleic acid nanoprecipitate that have also been developed using nanoprecipitation process in our lab [21-23, 28, 30-34]. Nano-FdUMP illustrated nanoscale particle size $(\sim 35 \mathrm{~nm}$, polydispersity index $\approx$ $0.3)$ and neutral surface charge $(\sim 2 \mathrm{mV})$ (fig. 1c). The encapsulation efficiency (EE \%) and loading capacity (LC $\%)$ of FdUMP in Nano-FdUMP were $~ 98 \%$ and $~ 38$ wt $\%$, respectively, as measured using HPLC, which were

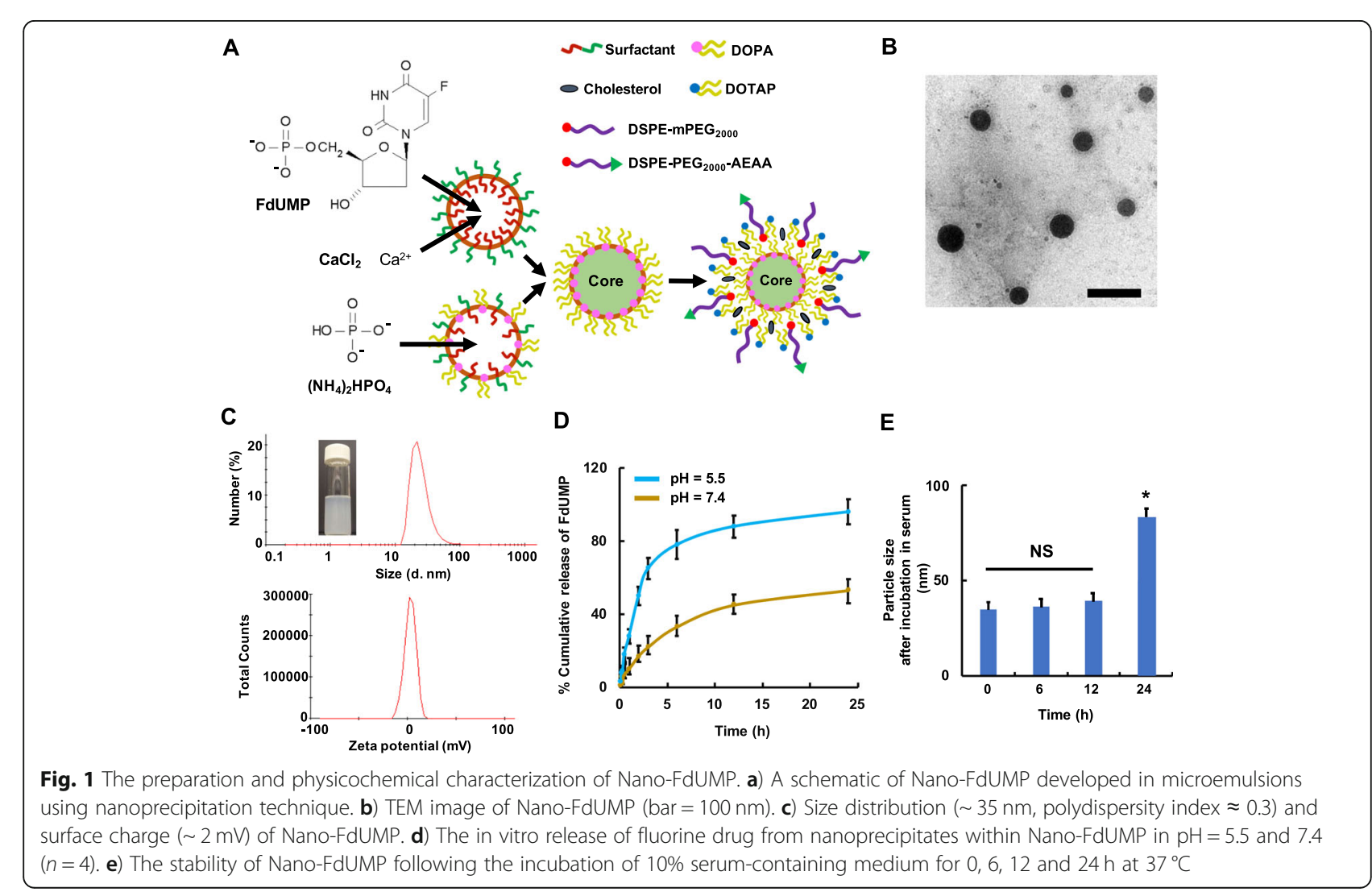


similar to EE \% and LC \% for FdUMP in Nano-FdUMP without AEAA. As shown in fig. 1d, $>95 \%$ of FdUMP were released from the nanoprecipitate inside NanoFdUMP when incubated within acidic PBS for $24 \mathrm{~h}$, which was significantly more efficient than the drug release in neutral PBS. These indicate that Nano-FdUMP showed $\mathrm{pH}$-sensitive drug release, which is most likely due to the acid-sensitive feature of $\mathrm{Ca}_{3}\left(\mathrm{PO}_{4}\right)_{2}$ [35]. Nano-FdUMP maintained in vitro stability in serumcontaining medium up to $12 \mathrm{~h}$, but significant aggregation occurred at $24 \mathrm{~h}$ (fig. 1e). In addition, Nano-FdUMP without AEAA demonstrated similar morphology, particle size, surface charge, drug release and serum stability (Fig. S1) as observed for Nano-FdUMP (fig. 1).

Recently, several nanoformulations have been developed for delivery of 5 -Fu in tumor-bearing mouse models [36-38]. For example, $\mathrm{Li}$ et al. produced a poly( $\gamma$-benzyl-L-glutamate)-based NP for delivery of 5$\mathrm{Fu}$ in subcutaneous CRC mouse model; however, EE\% and LC\% were only $\sim 61 \%$ and $\sim 27 \%$, respectively [36]. Safwat and colleagues also developed a gold NP-based system for delivery of 5-Fu in skin cancer mouse model, but $\mathrm{EE} \%$ was less than 70\% (LC\% was not mentioned) [37]. In addition, Kazi and coworkers designed a poly (lactic-co-glycolic acid)-based NP for delivery of 5-Fu in melanoma mouse model, but $\mathrm{EE} \%$ and $\mathrm{LC} \%$ were only $\sim 56 \%$ and $\sim 2 \%$, respectively [38]. In the present study, Nano-FdUMP achieved significantly higher EE\% ( 98\%) and LC\% ( 38\%) of fluorine drug than these previously reported studies.

It is known that 5-Fu can be metabolized into FdUMP within cancer cells, and FdUMP forms a complex with thymidylate synthase for inhibition of deoxythymidine monophosphate (dTMP) production [19]. However, intracellular metabolism of 5-Fu into FdUMP is a rate-limiting process that dampens therapeutic efficacy; for example, over $80 \%$ of a single dose of $5-\mathrm{Fu}$ is converted to inactive metabolites [39]. In addition, although 5 -Fu is well tolerated, serious toxic signs are found in patients who have deficiency of dihydropyrimidine dehydrogenase, an enzyme that is responsible for metabolism of $5-\mathrm{Fu}$. This toxicity is due to 5-Fu but not metabolites [39]. In order to bypass these resistances, FdUMP, instead of 5 - Fu, was formulated using our AEAA-targeted PEGylated NP (Nano-FdUMP) (fig. 1a). Free FdUMP, being a nucleoside phosphate, is impermeable into cells [40], while Nano-FdUMP can efficiently carry the impermeable FdUMP into cancer cells (see below results).

Taken together, Nano-FdUMP potentially provides the advantages over the previously reported 5-Fu nanoformulations, from the encapsulation efficiency/ loading capacity and the mechanism of action points of view.

\section{In vitro anticancer effects of nano-FdUMP}

Nano-FdUMP caused significantly higher cytotoxicity $\left(\mathrm{IC}_{50} \approx 20 \mu \mathrm{M}, 24 \mathrm{~h}\right.$ incubation; $p<0.01$ ) in mouse CRC (CT26) and HCC (Hepa1-6) cell lines relative to 5-Fu $\left(\mathrm{IC}_{50} \approx 70 \mu \mathrm{M}, 24 \mathrm{~h}\right.$ incubation) (fig. 2a). Nano-dUMP, in which FdUMP was replaced by 2 '-deoxyuridine $5^{\prime}$ monophosphate (dUMP), was chosen as negative control. Of note, $\mathrm{IC}_{50}$ of Nano-dUMP could not be determined under the conditions tested, demonstrating that neither dUMP nor AEAA-targeted formulation was cytotoxic. In addition, no significant difference in apoptosis of CT26 and Hepa1-6 cells was observed between Nano-dUMP and PBS (fig. 2b), while Nano-FdUMP induced significantly higher level of apoptosis $(p<0.01,24$ $\mathrm{h}$ incubation) as compared to Nano-dUMP and 5-Fu (fig. 2b). These indicate that cytotoxic and apoptotic effects of Nano-FdUMP were mainly due to delivery of fluorine drug using AEAA-targeted nanoformulation.

It has been reported that the increment of ROS is associated with the progress of apoptosis [41]. The capacity of Nano-FdUMP to induce ROS was subsequently assessed in CT26 and Hepa1-6 cells (fig. 2c). Results showed that no significant difference in ROS formation was found in cancer cells between PBS and NanodUMP, while the ROS was significantly induced by 5 -Fu $(p<0.05,24 \mathrm{~h})$. Furthermore, Nano-FdUMP caused significantly higher level of ROS $(p<0.01,24 \mathrm{~h})$ than 5 -Fu (fig. 2c). Glutathione (GSH) is known as the primary endogenous antioxidant, and plays a key role in neutralization of intracellular ROS by direct and indirect scavenging [42]. As the synthesis of GSH is mainly relied on L-cysteine [43], and N-acetyl-L-cysteine (NAC) is the acetylated variant (a precursor) of L-cysteine [44], NAC can be used to provide L-cysteine for GSH production. Here, NAC was used to investigate the role of ROS achieved by Nano-FdUMP in the induction of apoptosis (fig. 2d). When cancer cells were pretreated with NAC, the apoptotic efficacy of Nano-FdUMP was significantly reduced $(p<0.05,24 \mathrm{~h})$ from $\sim 30 \%$ to $\sim 15 \%$ (Fig. $2 \mathrm{~d}$ ). These results showed that the apoptosis of CRC and HCC cells is, at least, in part due to ROS formation achieved by Nano-FdUMP.

\section{In vivo profiles of nano-FdUMP}

The in vivo toxicity of Nano-FdUMP was first assessed in healthy mice (Fig. S2). No significant loss of body weight was found in Nano-FdUMP at 5,10 and $25 \mathrm{mg} /$ $\mathrm{kg}$ FdUMP; however, Nano-FdUMP at $50 \mathrm{mg} / \mathrm{kg}$ of FdUMP caused slight body weight loss (Fig. S2). In addition, toxic signs (e.g., hunched posture, ruffled hair coat, and reluctance to move) were observed in mice treated with Nano-FdUMP at higher dose $(50 \mathrm{mg} / \mathrm{kg})$ but not at lower doses (5, 10 and $25 \mathrm{mg} / \mathrm{kg}$ ) (Fig. S2). 

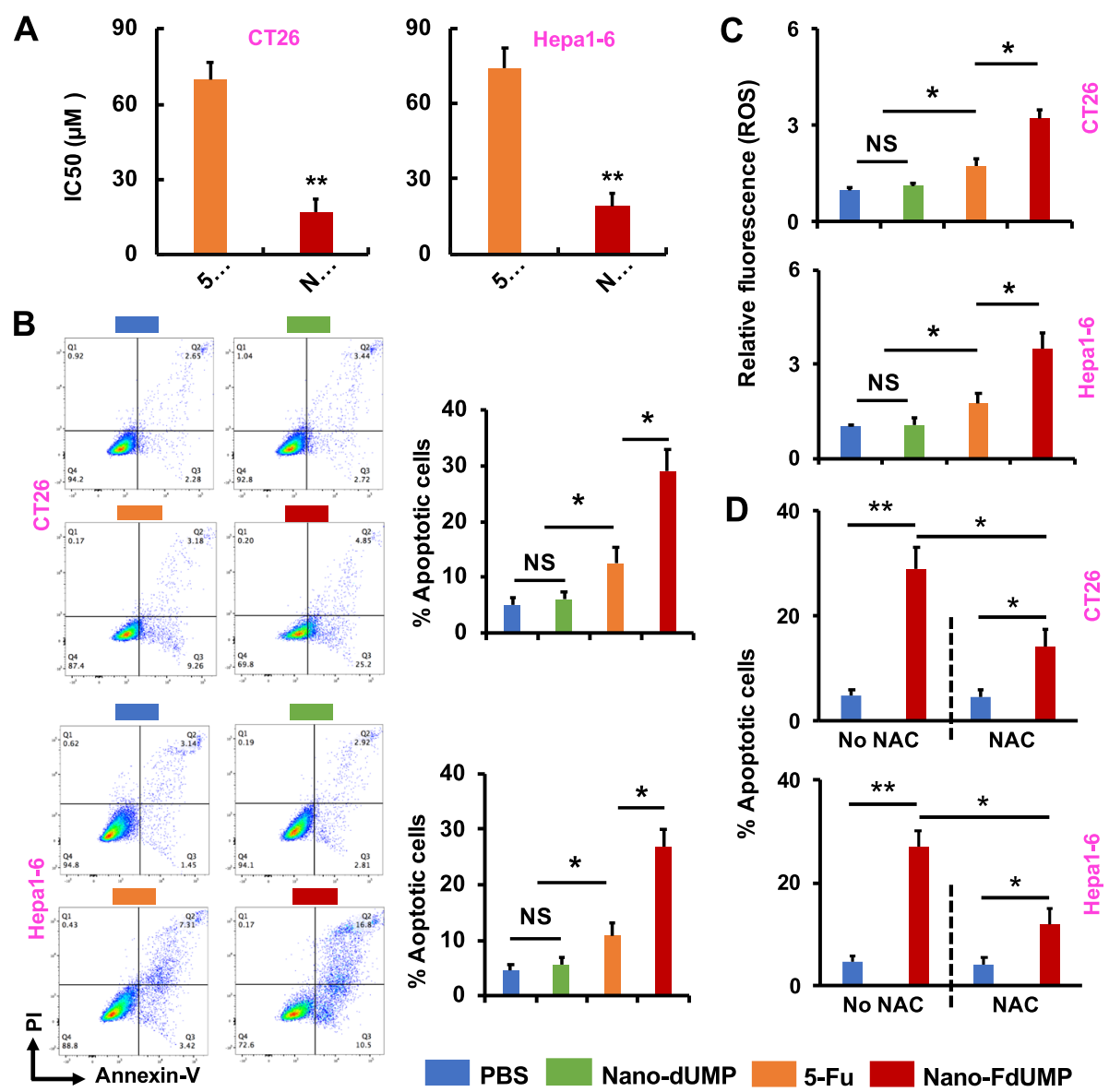

Fig. 2 In vitro studies of Nano-FdUMP. a) Cytotoxicity of CT26 and Hepa1-6 cells treated with 5-Fu and Nano-FdUMP $\left(n=3,{ }^{* *} p<0.01\right)$. b) Apoptotic CT26 and Hepa1-6 cells (\%) treated with PBS, 5-Fu, Nano-dUMP and Nano-FdUMP were measured by Annexin V-FTIC and PI assay $\left(n=3,{ }^{*} p<0.05\right)$. c The ROS level in CT26 and Hepa1-6 cells treated with PBS, 5-Fu, Nano-dUMP and Nano-FdUMP ( $\left.\mathrm{n}=3,{ }^{*} p<0.05\right)$. d) Apoptotic CT26 and Hepa1-6 cells (\%) treated by Nano-FdUMP following incubation with or without NAC $\left(n=3,{ }^{*} p<0.05\right.$ and $\left.{ }^{* *} p<0.01\right)$

The i.v. administration of 5-Fu causes short blood circulation and quick systemic elimination [45]. PEGylated nanoformulation may significantly increase the half-life of chemotherapeutics in the bloodstream [46]. In this study, the half-life of Nano-FdUMP was determined using orthotopic CT26-FL3 derived CRC and Hepa1-6Luc derived HCC mouse models (fig. 3a). Results showed that the concentration of fluorine drug in the plasma decreased rapidly, and a minor level was detected at $1 \mathrm{~h}$ post injection $\left(\mathrm{t}_{1 / 2} \approx 6 \mathrm{~min}\right.$ and $5 \mathrm{~min}$ for 5 - $\mathrm{Fu}$ in CRC and HCC models; fig. 3a). In contrast, fluorine drug in Nano-FdUMP was more slowly eliminated from the plasma $\left(\mathrm{t}_{1 / 2} \approx 1.6 \mathrm{~h}\right.$ and $1.4 \mathrm{~h}$ for FdUMP in CRC and HCC models; fig. 3a). In addition, Nano-FdUMP without AEAA demonstrated similar half-lives (Fig. S3) as recorded by Nano-FdUMP with AEAA (fig. 3a). These results confirmed that the half-life of fluorine drug was significantly improved by Nano-FdUMP.

The tissue distribution of Nano-FdUMP was also investigated using orthotopic CRC and HCC mouse models.
Following i.v. injection of DiD-labeled nanoformulations, tumors and major tissues were ex vivo imaged using the IVIS $^{\odot}$ Kinetics Optical System (fig. 3b and c). In CRC model, AEAA-targeted Nano-FdUMP achieved significantly higher retention in the tumor $(\sim 2.5$ fold; $p<0.05)$ but significantly less accumulation in the liver $(\sim 2$ fold; $p<0.05)$ than non-targeted nanoformulation (fig. 3b). In HCC model, AEAA-targeted nanoformulation was mainly accumulated inside liver tumor, which was confirmed by the colocalization of NPs (fluorescence imaging from DiD dye) and tumor tissue (bioluminescence imaging from visible light produced by luciferase in tumor cells) (fig. 3c). However, non-targeted nanoformulation was mainly found in healthy liver rather than the tumor (fig. 3c). It is known that AEAA is a high affinity ligand $(\mathrm{Kd} \approx 9 \mathrm{nM})$ for the sigma-1 receptor which is expressed in most of solid tumor cells (e.g., CT26 and Hepa1-6 cells) [7, 24]. Results in fig. $3 \mathrm{~b}$ and $\mathrm{c}$ confirmed that AEAA-targeted nanoformulation significantly improved tumor accumulation and alleviated non-specific tissue distribution. Due to $\mathrm{pH}$ - 


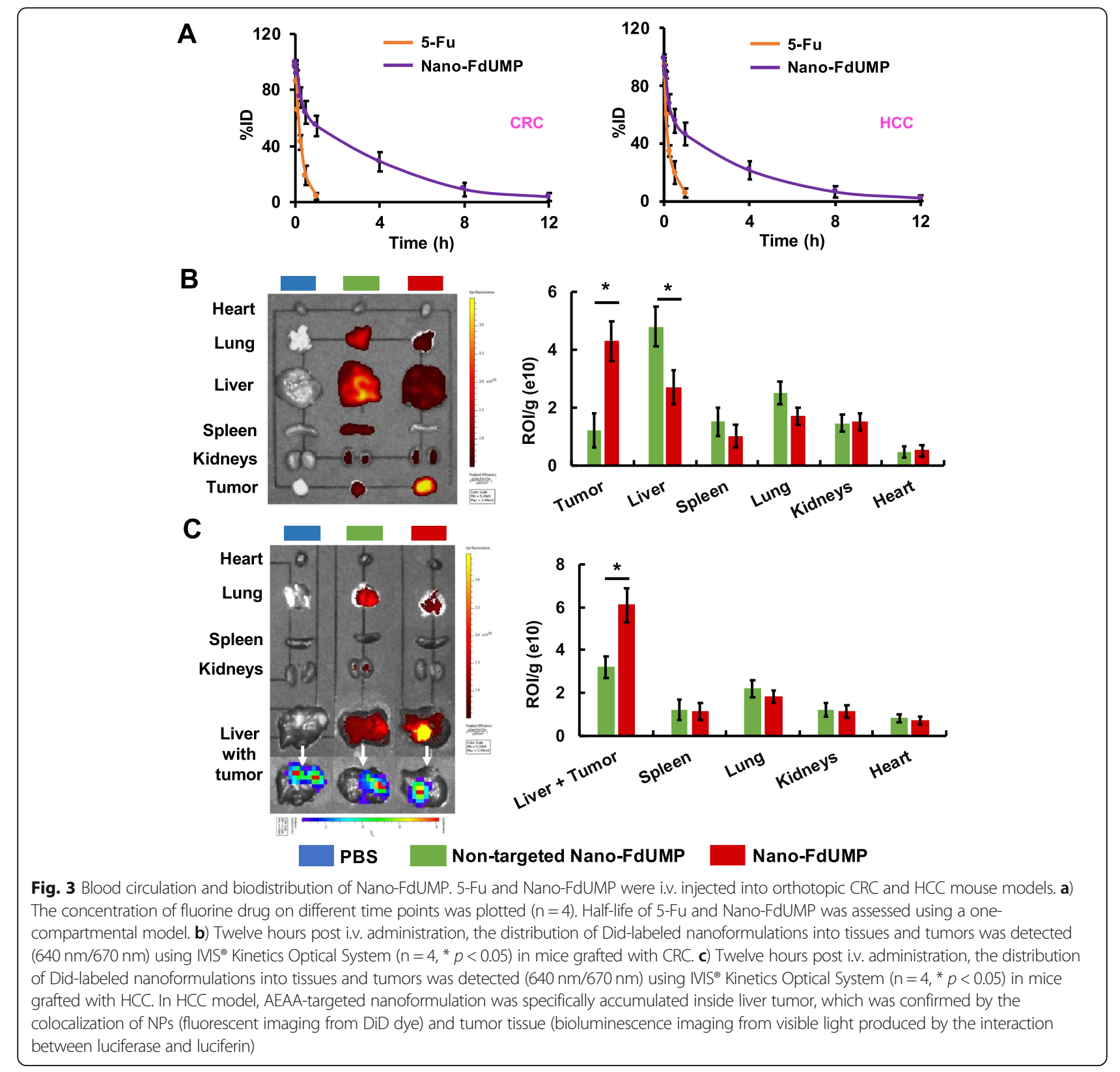

sensitive property (fig. 1d), fluorine drug will be released from AEAA-targeted nanoformulation inside the tumor (see below discussion), which is reminiscent of NanoFolox that could also specifically achieve the delivery and release of platinum drug and FnA inside the tumor [7].

Furthermore, the antitumor efficacy of Nano-FdUMP was assessed in orthotopic CT26-FL3 derived CRC and Hepa1-6-Luc derived HCC mouse models (Fig. S4). No significant antitumor efficacy was achieved by 5 - $\mathrm{Fu}(50$ $\mathrm{mg} / \mathrm{kg}$ ) as compared to PBS, while the growth of CRC and HCC was significantly slowed down by NanoFdUMP (10 and $25 \mathrm{mg} / \mathrm{kg}$ ) (Fig. S4), indicating that Nano-FdUMP at lower doses could achieve significantly improved therapeutic efficacy as compared to $5-\mathrm{Fu}$ at higher doses. In addition, no significant antitumor efficacy was achieved by non-targeted Nano-FdUMP as compared to PBS, but AEAA-targeted Nano-FdUMP significantly retarded tumor growth $(p<0.05)$ than nontargeted nanoformulation (Fig. S5), confirming AEAAmediated antitumor effect.

\section{In vitro synergistic ICD effects of nano-FdUMP and nano- Folox}

It is well established that ICD-associated immunogenicity can be evoked by ROS [15], and the efficacy of ICD may be improved by ROS-inducing strategies [16-18]. It 
was previously reported by our laboratory that NanoFolox resulted in OxP-mediated ICD for anticancer immune response [7]. Here, synergistic ICD effects of Nano-FdUMP and Nano-Folox were assessed using CT26 and Hepa1-6 cells in terms of ICD hallmarks, namely exposure of calreticulin (CRT), secretion of adenosine triphosphate (ATP), and release of high mobility group protein B1 (HMGB1) [15].

Results in fig. 4a show that no significant difference in exposure of CRT was observed between Nano-FdUMP and PBS, most likely due to the inefficiency of 5-Fu or metabolites in facilitating the translocation of CRT [47]. In contrast, Nano-Folox was able to mediate significantly efficient CRT exposure $(p<0.01, \sim 31$ to $32 \%)$ onto the cell membrane (fig. 4a), which has been previously reported [7]. Notably, combination of Nano-FdUMP and Nano-Folox further improved translocation of CRT $(p<$
$0.05, \sim 73$ to $79 \%)$ relative to either nanoformulations (fig. 4a).

Although 5-Fu or metabolites cannot effectively induce the CRT exposure, they may facilitate the ATP secretion and HMGB1 release [47]. Indeed, Nano-FdUMP significantly activated the secretion of ATP into the extracellular milieu of cancer cells $(p<0.05)$ as compared to PBS, which were similar to results obtained by Nano-Folox (fig. 4b). Of note, the combination of two nanoformulations further enhanced the secretion of ATP $(p<0.01)$ relative to either nanoformulations (fig. 4b).

Moreover, Nano-FdUMP significantly exerted the release of HMGB1 from the nucleus into the extracellular milieu of cancer cells as compared to PBS, which were similar to results found in Nano-Folox (fig. 4c). The combination of two nanoformulations further promoted the release of HMGB1 $(p<0.05)$ relative to either

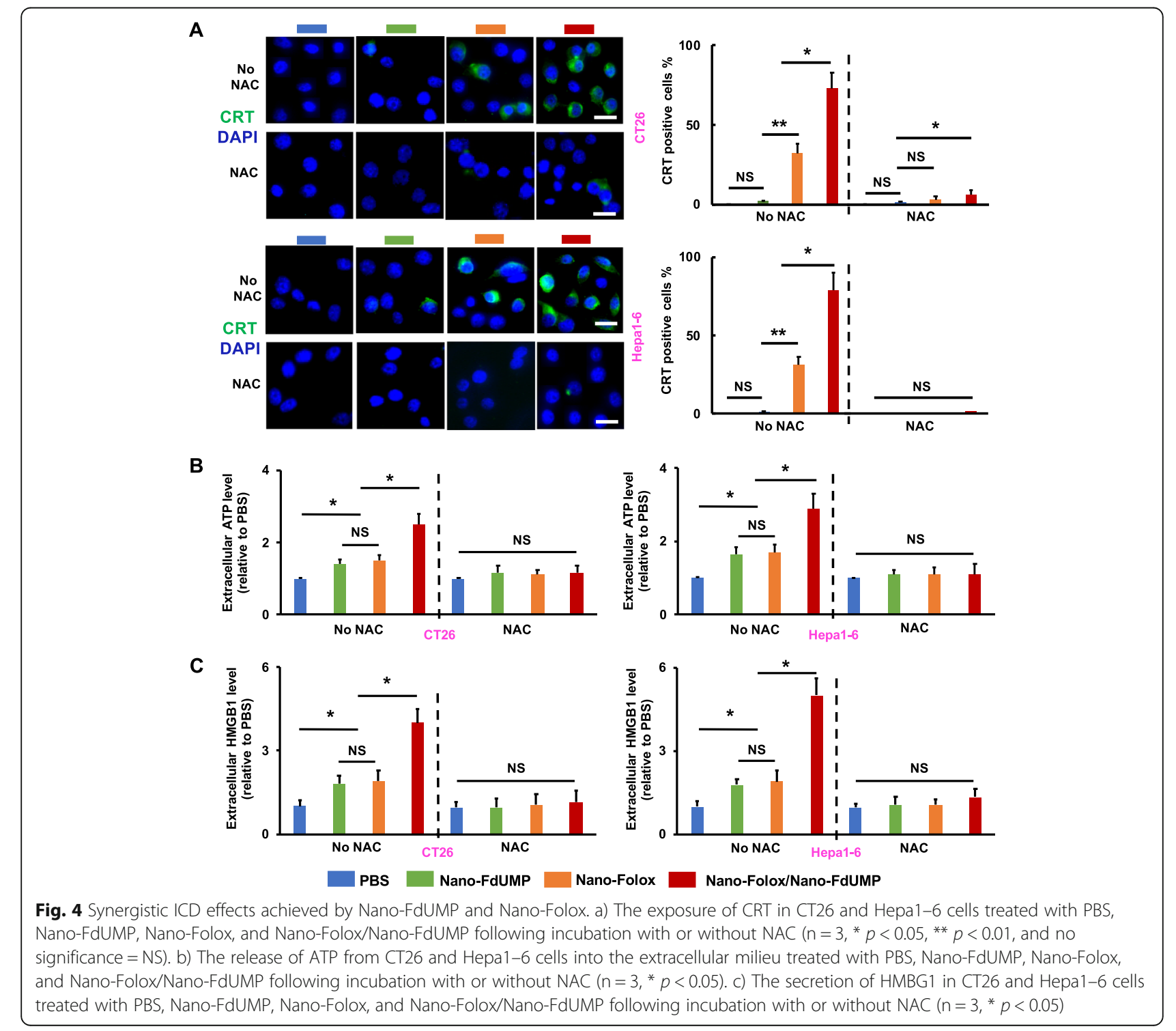


nanoformulations (fig. 4c). These results demonstrated that Nano-FdUMP was not able to induce ICD efficacy on its own, but could synergize with Nano-Folox for improved ICD effects.

It is worth noting that when cancer cells were pretreated with NAC, the activity of ICD hallmarks was significantly suppressed in either Nano-FdUMP, NanoFolox, or combination (fig. 4), indicating that 1 ) the production of ROS is critical for Nano-Folox-mediated ICD induction, most likely due to the fact that OxP induces ICD via both endoplasmic reticulum (ER) stress and ROS generation; 2) the critical role of ROS achieved by Nano-FdUMP in promoting ICD effects of Nano-Folox.

\section{Combination of nano-FdUMP and nano-Folox for synergistic chemo-immunotherapy in orthotopic CRC and HCC mouse models}

Due to in vitro synergistic ICD efficacy achieved by Nano-FdUMP and Nano-Folox, the combination therapy of two nanoformulations was further evaluated in vivo using orthotopic CT26-FL3 derived CRC and Hepa1-6Luc derived HCC mouse models (figs. 5 and 6). Based on results of Fig. S4, Nano-FdUMP containing $10 \mathrm{mg} / \mathrm{kg}$ of FdUMP was chosen for in vivo combination therapy. Based on results of fig. $3 \mathrm{~b}$ and $\mathrm{c}$ and fig. S5, nontargeted Nano-FdUMP was not used for in vivo combination therapy. In addition, "Nano-Folox and free 5-Fu" has demonstrated significantly improved therapeutic outcome than FOLFOX (free drugs) [7]. Thus, "NanoFolox and free 5-Fu" was chosen as positive control in this study.

As shown in fig. 5a and b, combination of NanoFdUMP (10 mg/kg of FdUMP) and Nano-Folox (1.5 mg/ $\mathrm{kg}$ of platinum drug and $4.5 \mathrm{mg} / \mathrm{kg}$ of $\mathrm{FnA}$ ) significantly improved the antitumor efficacy than Nano-FdUMP alone, Nano-FdUMP with OxP (3 mg/ $\mathrm{kg}$ of platinum drug) and FnA $(90 \mathrm{mg} / \mathrm{kg}$ ), and Nano-Folox with 5 -Fu $(50 \mathrm{mg} / \mathrm{kg})$. Consequently, the combination of NanoFdUMP and Nano-Folox provided long-term survival in 5 out of 6 mice, which was significantly improved $(p<$ 0.001 ) than PBS [median survival $(\mathrm{MS})=40$ days $)$ ], Nano-FdUMP (MS $=45$ days), Nano-FdUMP with OxP and FnA (MS $=58$ days), and Nano-Folox with 5-Fu $(\mathrm{MS}=64$ days) (fig. 5c).

Nano-Folox was reported to cause platinum-DNAadducts for apoptosis, and the apoptotic efficacy was further enhanced when combined with 5-Fu [7]. In this study, immunofluorescence results showed that combination of Nano-FdUMP and Nano-Folox significantly $(p<0.05)$ induced apoptosis in tumors $(\sim 32 \%)$ relative to PBS $(\sim 0.3 \%)$, Nano-FdUMP alone $(\sim 2 \%)$, NanoFdUMP with OxP and 5-Fu $(\sim 4 \%)$, and Nano-Folox with 5-Fu ( 9\%) (fig. 5d). The apoptotic efficacy was also confirmed by the detection of cleaved (activated) caspase 3 (fig. S6). The enhanced apoptotic efficacy is most likely due to the fact that 1 ) the efficacy of 5 -Fu metabolite was promoted by FnA released from NanoFolox; 2) 5-Fu metabolite/FnA further enhanced apoptotic effect with OxP derivative released from NanoFolox.

The combination of two nanoformulations induced a shift from a "cold" tumor microenvironment (TME) into a "hot" one (fig. 5e and f). It was supported by the increment of immunostimulatory factors and the reduction of immunosuppressive factors. For example, $\mathrm{CD}^{+} \mathrm{T}$ cells, $\mathrm{CD}^{+} \mathrm{T}$ cells and dendritic cells (DCs) were significantly activated in tumors by the combination strategy (fig. 5e), which were accompanied with the upregulation of IFN$\gamma$, TNF- $\alpha$ and IL-12, three cytokines for the activation of antitumor immunity (fig. 5f) [48]. On the contrary, myeloid derived suppressor cells (MDSCs), regulatory T cells (Tregs) and tumor-associated macrophages (M2) were significantly decreased in tumors by the combination strategy (fig. 5e), which were accompanied with downregulation of immunosuppressive cytokines such as IL-4, IL-6 and IL-10 (fig. 5f).

The ICD-associated antitumor immunity is essentially relied on the activation of effector $\mathrm{T}$ cells for killing tumor cells [17]. The orthotopic CRC animals were administrated with Nano-FdUMP/Nano-Folox following the depletion of either $\mathrm{CD}^{+}$or $\mathrm{CD} 4^{+} \mathrm{T}$ cells with corresponding monoclonal anti-CD8 or -CD4 antibody (fig. $5 \mathrm{~g})$. Consequently, the antitumor efficacy of NanoFdUMP/Nano-Folox was significantly suppressed $(p<$ 0.01 ) following the injection of these antibodies, but not the isotype IgG (fig. 5g), These results confirmed the critical role of effector $\mathrm{T}$ cells for antitumor immunity mediated by the combination strategy.

It has been reported that FOLFOX has demonstrated great potential for the generation of memory $\mathrm{T}$ cells [49], and IL-12 plays key role in the activation and proliferation of antigen-specific memory $\mathrm{T}$ cells $[50,51]$. Indeed, memory $\mathrm{CD}^{+}$and $\mathrm{CD}_{4}^{+} \mathrm{T}$ cells were successfully activated in tumors following treatment of NanoFdUMP/Nano-Folox (fig. 5e). In order to confirm tumor-specific memory response, tumor-free mice "cured" by the treatment of Nano-FdUMP/Nano-Folox were rechallenged with $4 \mathrm{~T} 1$ and CT26-FL3 cells (Fig. S7). Results showed that $4 \mathrm{~T} 1$ breast tumor growth was not affected, while CT26-FL3 tumor growth was significantly inhibited in same animals (Fig. S7A). These results confirmed that the combination approach promises for the induction of tumor-specific memory response against CRC, resulting in long-term survival in orthotopic CRC mice (fig. 5c).

In addition, significantly improved antitumor efficacy was also achieved by the combination strategy in orthotopic HCC mice as compared to the other controls 


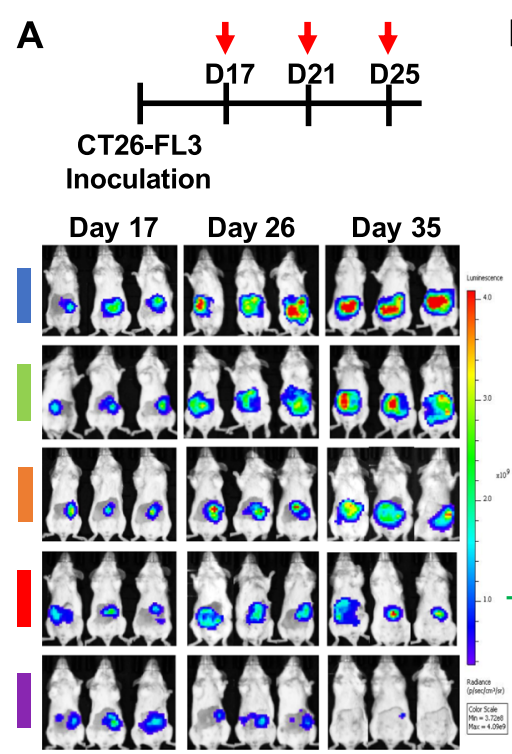

B

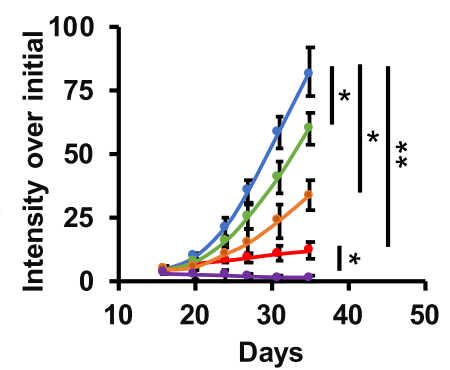

D

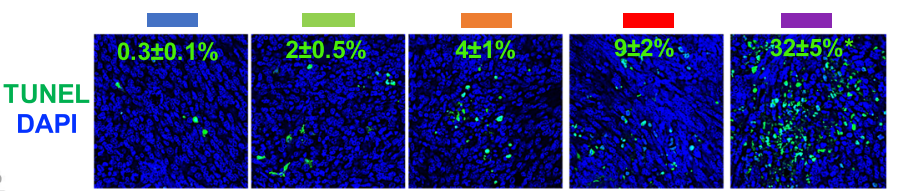

E

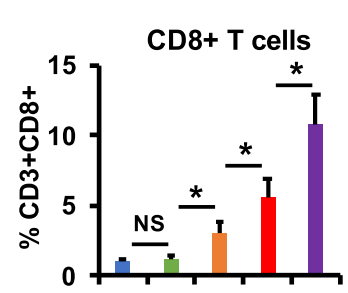

Activated DCs

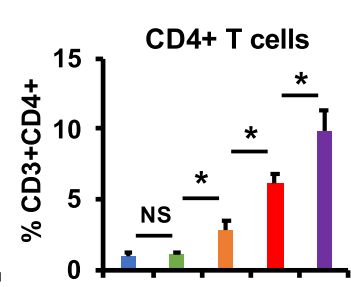

MDSCs
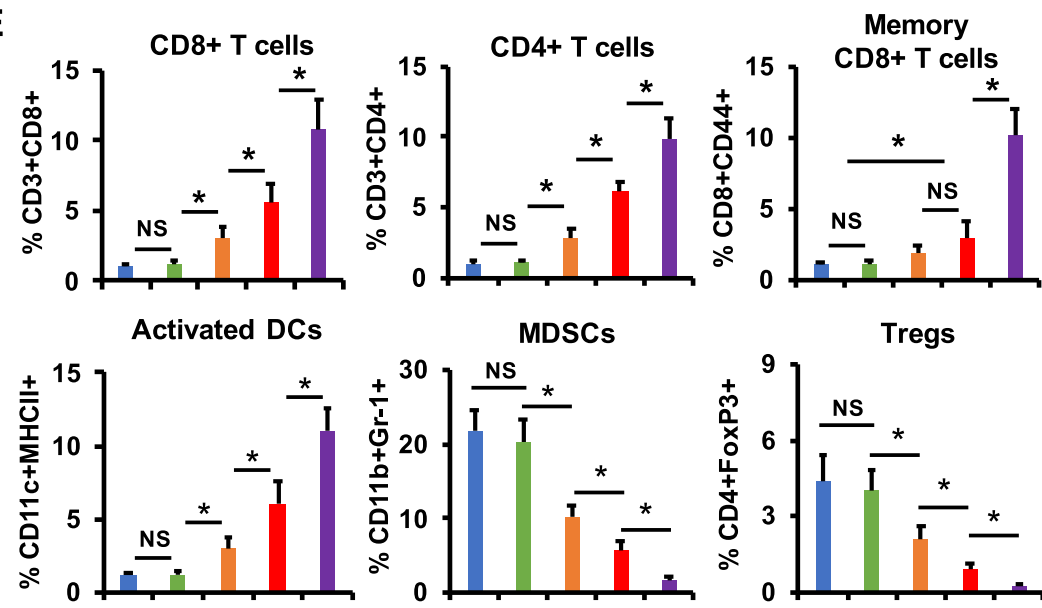

Tregs

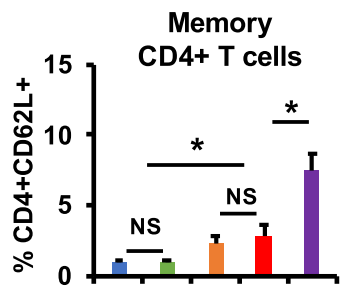

M2 cells
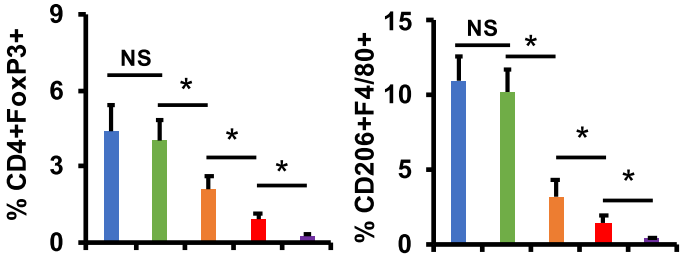

G Nano-Folox/Nano-FdUMP

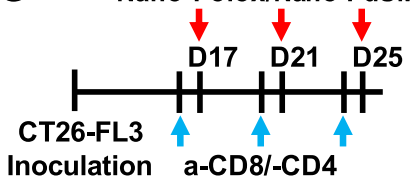

Inoculation a-CD8/-CD4

PBS

a-CD8+Nano-Folox/Nano-FdUMP

a-CD4+Nano-Folox/Nano-FdUMP IgG+Nano-Folox/Nano-FdUMP

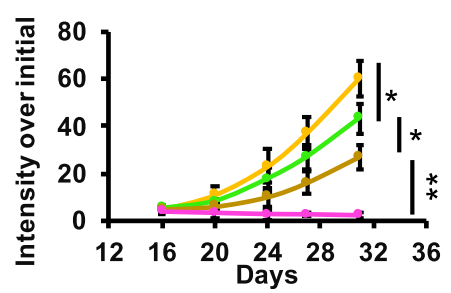

Fig. 5 (See legend on next page.) 
(See figure on previous page.)

Fig. 5 Chemo-immunotherapeutic effects of two nanoformulations in orthotopic CRC mouse model. a) Treatment schedule and IVIS images. b) The CT26-FL3 tumor growth over a 35-day period ( $n=6,{ }^{*} p<0.05$ and $\left.{ }^{* *} p<0.01\right)$. c) Animal survival (median survival: PBS $=40$ days, NanoFdUMP $=45$ days, Nano-FdUMP with OxP and FnA $=58$ days, and Nano-Folox with 5-Fu $=64$ days $)\left(n=6,{ }^{* * *} p<0.001\right)$. d) Immunofluorescent staining of tumors on Day 24 (DNA fragments = green; nuclei = blue) to determine apoptosis $\left(n=4,{ }^{*} p<0.05\right.$, relative to Nano-Folox/5-Fu). e) Level of $C D 8^{+} \mathrm{T}$ cells, CD4 ${ }^{+} \mathrm{T}$ cells, memory CD8 ${ }^{+} \mathrm{T}$ cells, memory CD4 ${ }^{+} \mathrm{T}$ cells, activated DCs, MDSCs, Tregs and M2 cells in tumors on Day 24 , analyzed by flow cytometry $\left(n=4,{ }^{*} p<0.05,{ }^{* *} p<0.01\right.$; NS $=$ no significance). $\left.\mathbf{f}\right)$ The mRNA expression of IFN- $\gamma$, TNF-a, IL-12, IL-4, IL-6 and IL-10 in tumors on Day $24\left(n=4,{ }^{*} p<0.05\right)$. g) Orthotopic CT26-FL3 tumor growth treated with Nano-FdUMP/Nano-Folox after the removal of CD4 ${ }^{+}$or CD8 ${ }^{+}$T cells $\left(n=4,{ }^{*} p<0.05\right.$ and $\left.{ }^{* *} p<0.01\right)$

(fig. 6a and b), which facilitated long-term survival in 4 out of 6 mice (fig. 6c). The chemo-immunotherapeutic effects including apoptosis (fig. 6d and Fig. S8) and TME remodeling (fig. 6e) were achieved by the combination strategy. The TME remodeling was supported by the increment of immunostimulatory factors and the reduction of immunosuppressive factors (fig. $6 \mathrm{e}$ and $\mathrm{f}$ ). Following treatment of Nano-FdUMP/Nano-Folox, $\mathrm{CD}^{+} \mathrm{T}$ cells, $\mathrm{CD} 4^{+} \mathrm{T}$ cells and $\mathrm{DCs}$ were significantly activated in tumors (fig. 6e), which were accompanied with increase of IFN- $\gamma$, TNF- $\alpha$ and IL-12 (fig. 6f). In contrast, MDSCs, Tregs and M2 cells were significantly decreased in tumors (fig. 6e), which were accompanied with alleviation of IL-4, IL-6 and IL-10 (fig. 6f). In addition, the antitumor efficacy of Nano-FdUMP/NanoFolox was also significantly suppressed in HCC mouse model following the pretreatment of anti-CD8 or antiCD4 antibodies (fig. $6 \mathrm{~g}$ ), confirming the critical roles of effector $\mathrm{T}$ cells for antitumor immunity mediated by the combination strategy. Furthermore, tumor-free mice "cured" by the combined approach were rechallenged with B16 melanoma and Hepa1-6-Luc cells (Fig. S7B). Results showed that B16 tumor growth was not affected in cured mice, while Hepa1-6-Luc tumor growth was significantly suppressed in same animals (Fig. S7B). These results showed that the combination approach also has the potential for the induction of tumor-specific memory response against $\mathrm{HCC}$, facilitating long-term survival in mice (fig. 6c).

In addition, no toxic signs were caused by the combination strategy as compared to PBS, which was confirmed by the analysis of body weight, hematological toxicity, and liver/kidney damage in healthy mice (Fig. S9). Taken together, the "Nano-FdUMP + Nano-Folox" strategy could achieve synergistic chemo-immunotherapeutic efficacy against CRC and HCC for long-term animal survival, without causing significant side effects.

Blockade of PD-L1 enhanced combination of nano-FdUMP and nano-Folox for inhibition of liver metastasis

FOLFOX has been used for patients with unresectable CRC liver metastases [3]; however, therapeutic outcome is still poor due to fast tumor progression. In this study, the "Nano-FdUMP + Nano-Folox" strategy was further applied to treat mice with experimental liver metastasis (fig. 7). This tumor-bearing model closely reproduces the aggressive pattern of CRC at metastatic stage [52]. As shown in fig. $7 \mathrm{a}$ and $\mathrm{b}$, the combined approach was able to significantly $(p<0.01)$ slow down tumor growth in mice as compared to PBS, which was accompanied by apoptosis (fig. $7 \mathrm{~d}$ and Fig. S10). In addition, NanoFdUMP/Nano-Folox significantly reprogrammed the TME in liver metastatic site (fig. 7e and f). However, no long-term survival ( $\mathrm{MS}=48$ days) was achieved by the Nano-FdUMP/Nano-Folox strategy after dosing (fig. 7c).

When combined with anti-PD-L1 mAb, NanoFdUMP/Nano-Folox significantly inhibited liver metastases $(p<0.01)$ as compared to either Nano-FdUMP/ Nano-Folox or anti-PD-L1 mAb (fig. 7a and b), which was accompanied with improved apoptosis (fig. $7 \mathrm{~d}$ and Fig. S10). Of note, combination of Nano-FdUMP/NanoFolox and anti-PD-L1 mAb was able to provide longterm survival in 5 out of 6 mice (fig. 7c). It is most likely due to the fact that combination of Nano-FdUMP/ Nano-Folox and anti-PD-L1 mAb significantly $(p<0.05$ and $p<0.01)$ increased the amount of effector/memory $\mathrm{T}$ cells and DCs (fig. 7e), upregulated the expression of IFN- $\gamma$ and IL-12 (fig. 7f) and reduced the level of IL-4, IL-6, and IL-10 (fig. 7f) in liver metastatic site, as compared to either FdUMP/Nano-Folox or anti-PD-L1 mAb. These indicated that FdUMP/Nano-Folox may significantly remodel the immunosuppressive TME for enhanced antitumor outcome in combination with immune checkpoint blockade, potentially providing a chemo-immunotherapeutic strategy for metastatic CRC.

\section{Discussion}

The development of nano delivery systems has significantly improved therapeutic efficacy and reduced side effects of anticancer agents [53]. However, the number of cancer nanomedicines approved for patients is still very few. Recently, four strategic directions have been proposed to foster nanomedicine translation and exploitation, including rational drug selection, combination therapy, immunotherapy, and patient stratification [54]. Our combination approach fulfills these directions, as follows: 
A

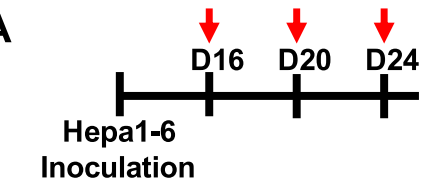

Day 16 Day 24 Day 32

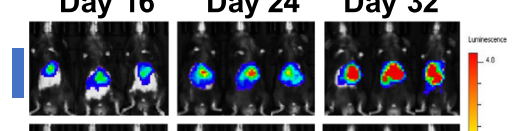

d)

Tim tol?
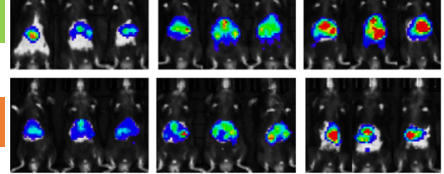

acialuas

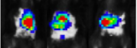

o i \& 8

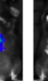

$\because 23$

2

E

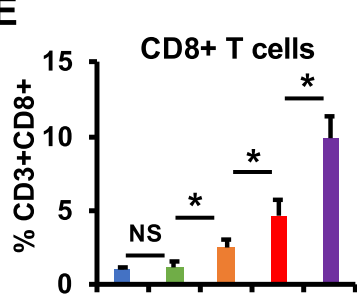

cis

$8 \%$

63
B

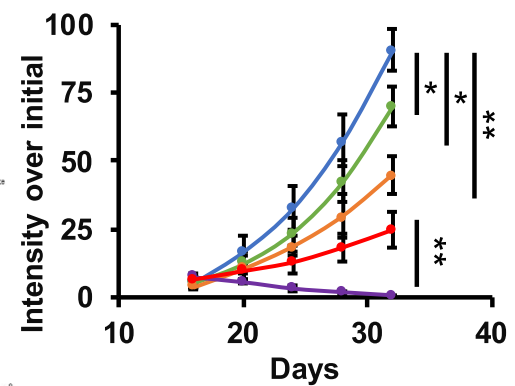

D

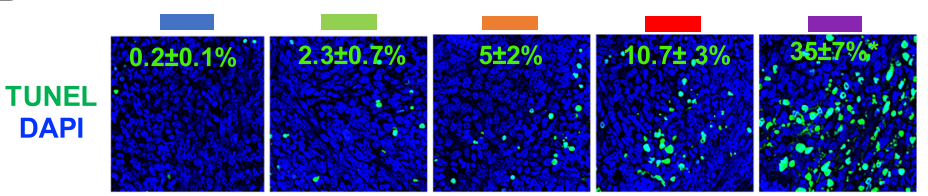

C

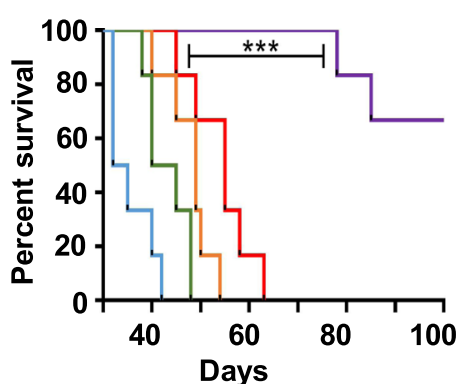

Memory CD8+ T cells
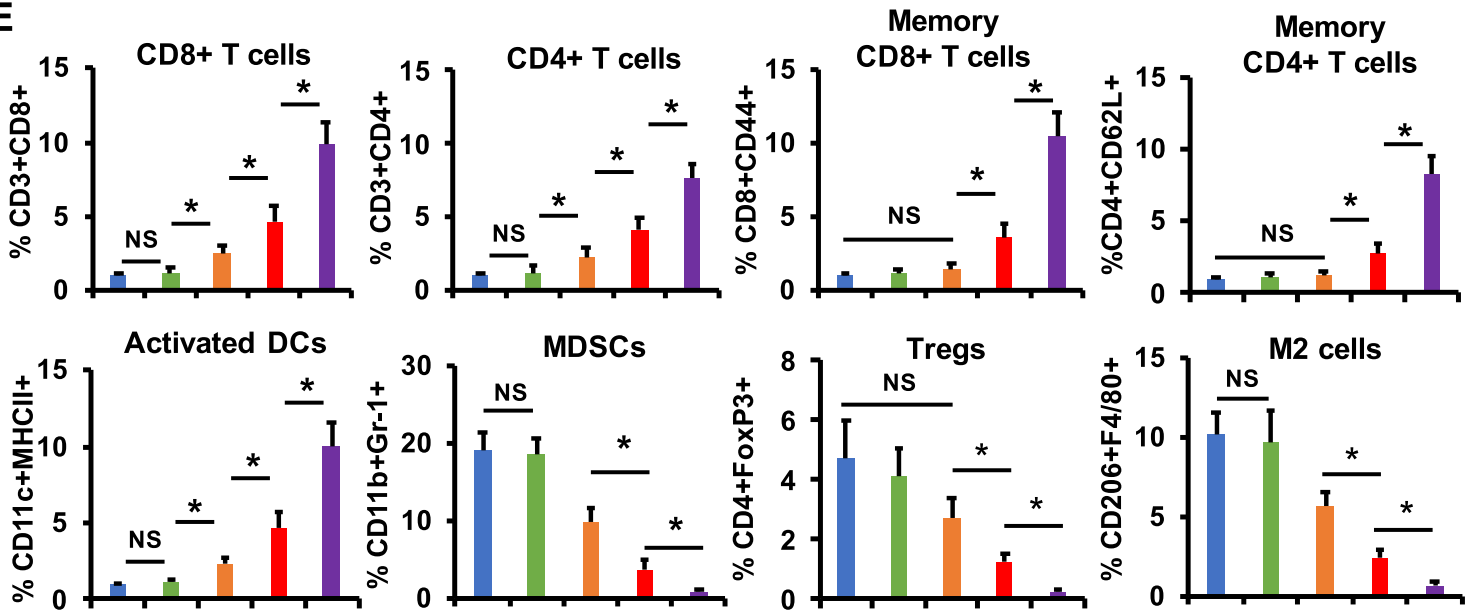

F

$F$ IFN-y TNF-a

IL-12

G

Nano-Folox/Nano-FdUMP

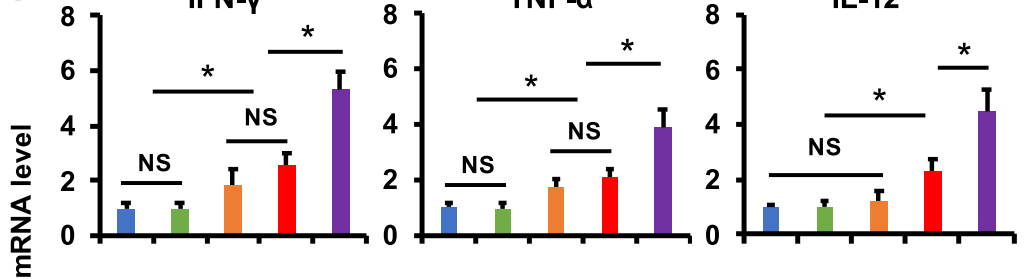

IL-10
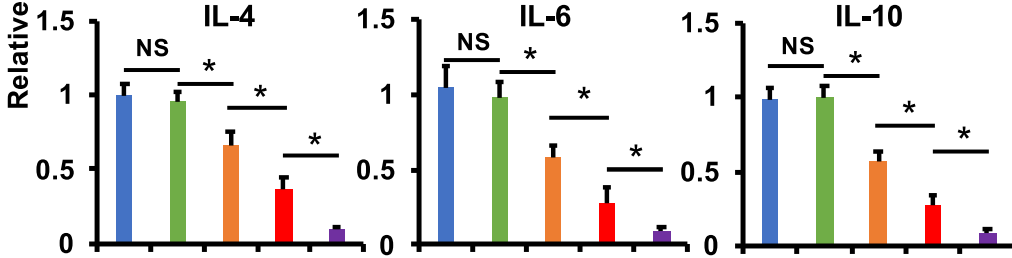

PBS Nano-FdUMP OxP/FnA/Nano-FdUMP

Nano-Folox/5-Fu Nano-Folox/Nano-FdUMP

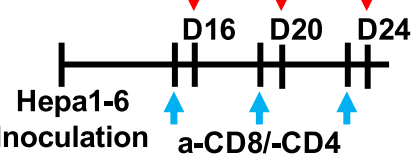

Inoculation a-CD8/-CD4

- PBS

- a-CD8+Nano-Folox/Nano-FdUMP

-a-CD4+Nano-Folox/Nano-FdUMP

- IgG+Nano-Folox/Nano-FdUMP

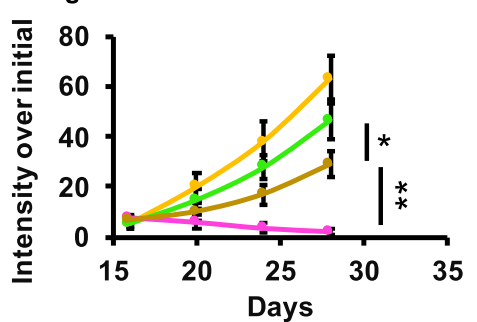

Fig. 6 (See legend on next page.) 
(See figure on previous page.)

Fig. 6 Chemo-immunotherapeutic effects of two nanoformulations in orthotopic HCC mouse model. a) Treatment schedule and IVIS images. b) The Hepa1-6-Luc tumor growth over a 32-day period $\left(n=6,{ }^{*} p<0.05\right.$ and $\left.{ }^{* *} p<0.01\right)$. c) Animal survival (median survival: PBS $=35$ days, NanoFdUMP $=41$ days, Nano-FdUMP with OxP and FnA $=49$ days, and Nano-Folox with 5-Fu $=55$ days $)\left(n=6,{ }^{* * *} p<0.001\right)$. d) Immunofluorescent staining of tumors on Day 23 (DNA fragments = green; nuclei = blue) to determine apoptosis $\left(\mathrm{n}=4,{ }^{*} p<0.05\right.$, relative to Nano-Folox/5-Fu). e) Level of CD8 ${ }^{+} \mathrm{T}$ cells, CD4 $4^{+} \mathrm{T}$ cells, memory CD8 ${ }^{+} \mathrm{T}$ cells, memory CD4 ${ }^{+} \mathrm{T}$ cells, activated DCs, MDSCs, Tregs and M2 cells in tumors on Day 23 , analyzed by flow cytometry ( $n=4,{ }^{*} p<0.05$; NS $=$ no significance). $\mathbf{f}$ ) The mRNA expression of IFN- $\gamma$, TNF- $\mathrm{a}, \mathrm{IL}-12, \mathrm{IL}-4$, IL-6 and IL-10 in tumors on Day $23\left(\mathrm{n}=4,{ }^{*} p<0.05\right.$; NS = no significance). g) Orthotopic Hepa1-6-Luc tumor growth treated with Nano-FdUMP/Nano-Folox after the removal of $\mathrm{CD}^{+}$or $\mathrm{CD}^{+} \mathrm{T}$ cells $\left(\mathrm{n}=4{ }^{*}{ }^{*} p<0.05\right.$ and $\left.{ }^{* *} p<0.01\right)$

1) Rational drug selection for chemo-immunotherapy. FOLFOX has been long utilized as the standard chemotherapy for CRC and HCC patients at advanced stages [1, 2]. It also demonstrates great potential for induction of robust antigen release from dying tumor cells and for generation of effector and memory $\mathrm{T}$ cells $[55,56]$. In this study, two AEAA-targeted PEGylated nanoformulations namely Nano-FdUMP (fig. 1) and Nano-Folox [7] were applied for FOLFOX-associated CRC and HCC therapies. Nano-FdUMP induced the formation of ROS (fig. 2), which significantly promoted Nano-Folox-mediated ICD efficacy (fig. 4). Consequently, synergistic chemo-
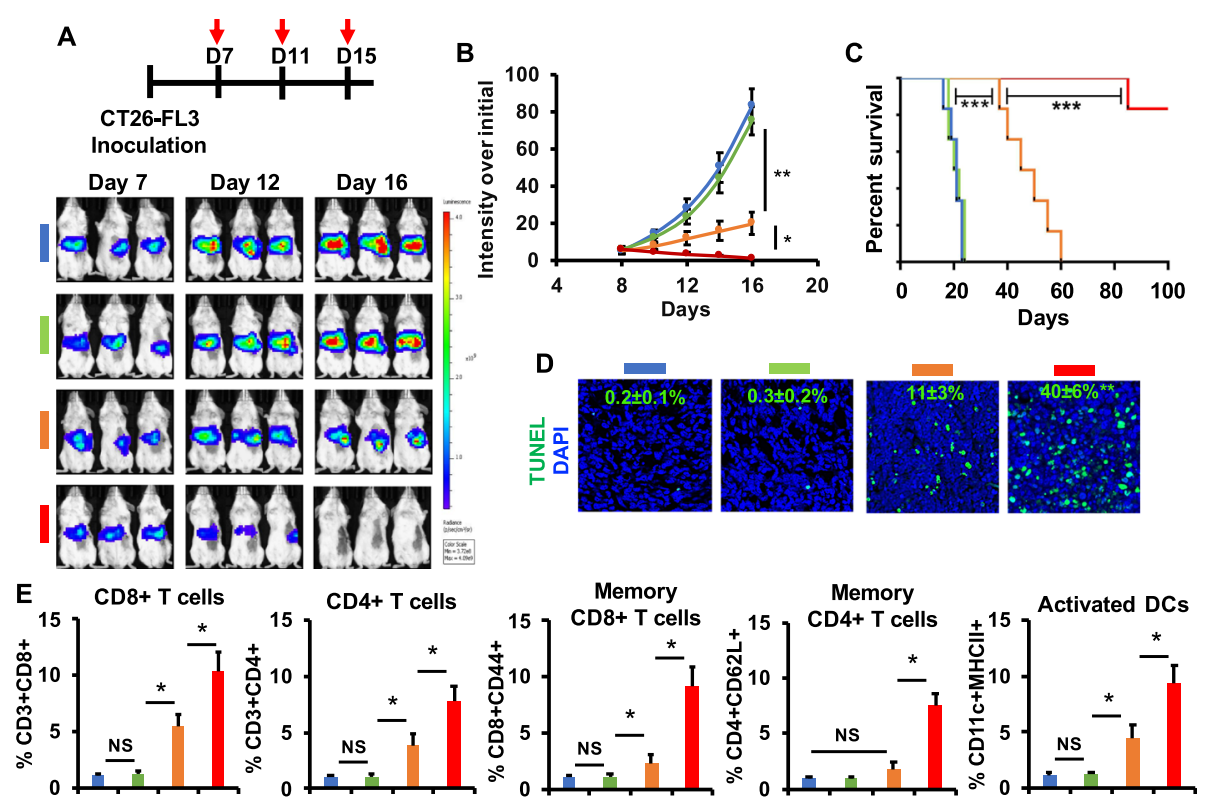

$\mathbf{F}$

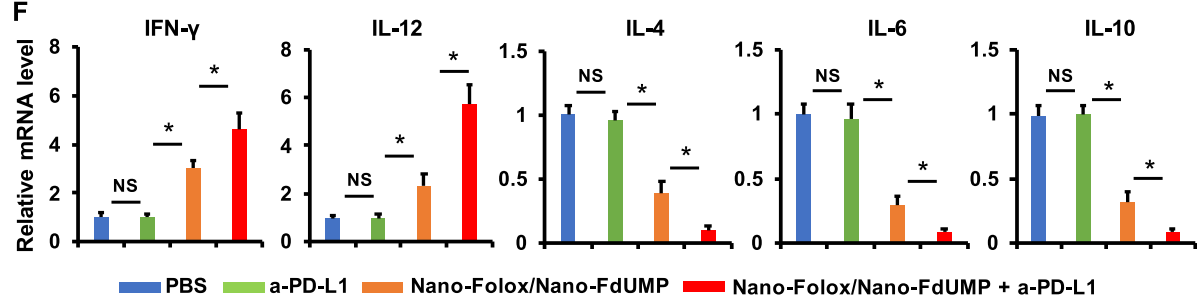

Fig. 7 The combination therapy of Nano-FdUMP/Nano-Folox and anti-PD-L1 antibody for CRC liver metastasis mouse model. a) Treatment schedule and IVIS images. b) The liver metastases over a 16-day period $\left(n=6,{ }^{*} p<0.05\right.$ and $\left.{ }^{* *} p<0.01\right)$. c) Animal survival (median survival: PBS $=20$ days, anti-PD-L1 antibody $=21$ days, and Nano-FdUMP/Nano-Folox $=48$ days) $\left(n=6,{ }^{* * *} p<0.01\right)$. d) Immunofluorescent staining of tumors on Day 12 (DNA fragments = green; nuclei = blue) to determine apoptosis $\left(n=4,{ }^{* *} p<0.01\right.$, relative to Nano-FdUMP/Nano-Folox). e) Level of $\mathrm{CD}^{+} \mathrm{T}$ cells, $\mathrm{CD} 4^{+} \mathrm{T}$ cells, memory $\mathrm{CD} 8^{+} \mathrm{T}$ cells, memory CD4 ${ }^{+} \mathrm{T}$ cells, and activated DCs in tumors on Day 12 , analyzed by flow cytometry ( $n=4,{ }^{*} p<0.05$ and NS = no significance). $\mathbf{f}$ ) The mRNA expression of IFN- $\mathrm{y}, \mathrm{IL}-12, \mathrm{IL}-4, \mathrm{IL}-6$ and IL-10 in tumors on Day 12 ( $n=4,{ }^{*} p<$ 0.05 and $\mathrm{NS}=$ no significance) 
immunotherapeutic efficacy was achieved in both orthotopic CRC and HCC mouse models using the "NanoFdUMP + Nano-Folox" strategy (figs. 5 and 6).

2) Combination therapy in microsatellite stable (MSS) CRC patients. Immune checkpoint inhibitors (e.g., antiPD-L1 mAb) have demonstrated efficacy in different cancers, but the response rate is still poor in CRC patients. Only a minor population of patients, who are diagnosed with microsatellite instable (MSI) CRC ( 15\% of total population) [57], respond to anti-PD-L1 mAb as a monotherapy [58]. It is now known that the immunosuppressive TME (also characterized as "cold" tumor) causes inefficiency of immune checkpoint inhibitors [59, $60]$. The shift of "cold" tumor to "hot" one potentially enhances the efficacy of checkpoint blockade [61]. In this study, the "Nano-FdUMP + Nano-Folox" strategy was able to induce ICD-associated antitumor immunity, which significantly reprogrammed immunosuppressive TME, improving antitumor efficacy against MSS CRC liver metastasis (established by CT26-FL3 cells, an MSS CRC cell line $[62,63])$ in combination with anti-PD-L1 $\mathrm{mAb}$ (fig. 7). Therefore, the combination of NanoFdUMP/Nano-Folox and anti-PD-L1 mAb will potentially achieve a superior outcome for CRC patients (particularly for MSS ones, up to $85 \%$ of total population) at advanced stages.

It is theoretically possible to encapsulate OxP, FnA and 5-Fu in a single nanoformulation. However, in comparison with the "all in one" nanoformulation, the "Nano-FdUMP + Nano-Folox" strategy holds greater potential for clinical translation, as follows:

1) Development of complex nanomedicines may not be beneficial for translation into clinical use [64, 65]. The "all in one" nanoformulation achieved using distinct functional materials may complicate large-scale manufacturing and cause unwanted toxic effects. In this study, Nano-Folox and Nano-FdUMP were achieved using nanoprecipitation process. It is a well-established formulation technique with biodegradable and biocompatible materials, and has been substantially utilized in our laboratory for delivery of chemotherapeutics and nucleic acids [21-23, 28, 30-34].

2) A variety of FOLFOX regimens are available in clinic such as FOLFOX-4, FOLFOX-6 and FOLFOX-7, and they differ in dose schedule of OxP and 5-Fu, and are chosen for cancer patients at different stages [66]. Our "Nano-FdUMP + Nano-Folox" strategy can be adjusted according to the clinical practice, while the adjustment in dose and timing of $\mathrm{OxP}$ and 5 - $\mathrm{Fu}$ is difficult in an "all in one" nanoformulation.

\section{Conclusions}

This study provides proof of concept that combination of two nano delivery systems may overcome the barriers associated with FOLFOX including non-specific delivery, high toxicity and long course of treatment, which can result in successful treatment for CRC and HCC. Further optimization in terms of dosing and timing will enhance clinical potential of our combination strategy for patients.

\section{Supplementary Information}

The online version contains supplementary material available at https://doi. org/10.1186/s12943-020-01297-0.

Additional file $\mathbf{1}$ Fig. S1. The physicochemical characterization of nontargeted Nano-FdUMP. A) TEM image (bar $=100 \mathrm{~nm}$ ). B) Size distribution $(\sim 38 \mathrm{~nm}$, polydispersity index $\approx 0.3)$ and surface charge $(\sim 5 \mathrm{mV})$. C) The in vitro release of fluorine drug from nanoprecipitates in $\mathrm{pH}=5.5$ and 7.4 $(n=4)$. d) No significant aggregation was caused in $10 \%$ serum-

containing medium up to $12 \mathrm{~h}$ at $37^{\circ} \mathrm{C}$. Fig. S2. Toxicity of Nano-FdUMP in healthy BALB/C mice. A) The body weight over a 35-day period following treatment of PBS and Nano-FdUMP containing 5, 10, 25 and $50 \mathrm{mg} /$ kg FdUMP on Day 1, 3 and 5. B) The overall condition of animals $(n=5)$ based on body condition scoring [BCS, IACUC Guidelines along with other criteria (e.g., hunched posture, ruffled hair coat, and reluctance to move)]. At the endpoint, the number of animals compliant with BCS index was presented. Results of non-targeted Nano-FdUMP were similar to those observed in targeted counterpart (Data not shown). Fig. S3. Blood circulation of non-targeted Nano-FdUMP in orthotopic CRC and HCC mouse models. Following i.v. injection, the concentration of fluorine drug on different time points was plotted $(n=4)$. Results showed that non-targeted Nano-FdUMP demonstrated similar blood circulation recorded by targeted counterpart (Fig. 3a). Fig. S4. Therapeutic efficacy of Nano-FdUMP in orthotopic CRC and HCC mouse models. Following treatment schedule as described in Figs. 5 and 6, Nano-FdUMP at doses of 10 and $25 \mathrm{mg} / \mathrm{kg}$ FdUMP achieved significantly improved antitumor efficacy as compared to PBS and $5-\mathrm{Fu}$ at $50 \mathrm{mg} / \mathrm{kg}\left(\mathrm{n}=5\right.$, ${ }^{*} p<0.05$ and $\left.p<0.01\right)$. Fig. S5. Therapeutic efficacy of Nano-FdUMP with/without AEAA at dose of $10 \mathrm{mg} / \mathrm{kg}$ FdUMP in orthotopic CRC and HCC mouse models. Following treatment schedule as described in Figs. 5 and 6, non-targeted NanoFdUMP could not slow down tumor growth as compared to PBS, but AEAA-targeted Nano-FdUMP achieved significantly improved antitumor efficacy than PBS and non-targeted Nano-FdUMP $\left(n=5,{ }^{*} p<0.05\right)$. Fig. S6. Immunofluorescent staining of tumors on Day 24 (as described in Fig. 5) (cleaved caspase 3 = green; nuclei = blue) to determine apoptosis $\left(n=3,{ }^{* *} p<0.01\right.$, relative to Nano-Folox/5-Fu). Fig. S7. Rechallenge studies. A) Orthotopic CRC mice (BALB/C) were treated with Nano-FdUMP/ Nano-Folox as described in Fig. 5, and one month after tumor disappearance, two flanks of mice were respectively rechallenged with $4 \mathrm{~T} 1$ and CT26-FL3 cells $\left(1 \times 10^{6}\right.$ cells per mouse) $(n=4)$. B) Orthotopic HCC mice (C57BL/6) were treated with Nano-FdUMP/Nano-Folox as described in Fig. 6 , and one month after tumor disappearance, two flanks of mice were respectively rechallenged with B16 and Hepa1-6-Luc cells $\left(1 \times 10^{6}\right.$ cells per mouse) $(n=4)$. Tumor volume was calculated using the formula $a^{2} b(\pi / 6)$, where $a$ is the minor diameter of the tumor and $b$ is the major diameter perpendicular to diameter a. Results confirmed that NanoFdUMP/Nano-Folox could induce tumor-specific memory response. Fig. S8. Immunofluorescent staining of tumors on Day 23 (as described in Fig. 6) (cleaved caspase $3=$ green; nuclei $=$ blue) to determine apoptosis ( $n=$ $3,{ }^{* *} p<0.01$, relative to Nano-Folox/5-Fu). Fig. S9. Toxicity studies of two nanoformulations in A) BALB/C and B) C57BL/6 mice. The body weight over a 35-day period following treatment of PBS and combination of two nanoformulations (Nano-Folox containing $1.5 \mathrm{mg} / \mathrm{kg}$ platinum drug was i.v. injected into mice on Day 1, 3 and 5. Eight hours post injection, Nano-FdUMP containing $10 \mathrm{mg} / \mathrm{kg}$ fluorine drug was i.v. injected into mice). Results show that no significant change was found in body weight and hematological/liver/kidney functions following treatment of two nanoformulations as compared to PBS $(n=5)$. Fig. S10. Immunofluorescent staining of tumors on Day 12 (as described in Fig. 7) (cleaved caspase $3=$ green; nuclei $=$ blue) to determine apoptosis $\left(n=3,{ }^{* *} p<\right.$ 
0.01, relative to Nano-FdUMP/Nano-Folox). Table S1. Antibodies used in the study. Table S2. Primers used for RT-PCR in the study.

\section{Abbreviations}

AEAA: Aminoethyl anisamide; ATP: Adenosine triphosphate; CRC: Colorectal cancer; CRT: Calreticulin; DAMPs: Damage-associated molecular patterns; DCs: Dendritic cells; DOPA: 1,2-dioleoyl-sn-glycero-3-phosphate; DOTAP: 1,2dioleoyl-3-trimethylammonium-propane; DSPE-PEG: N-(Carbonylmethoxypolyethyleneglycol 2000)-1,2-distearoyl-sn-glycero-3-

phosphoethanolamine; dTMP: Deoxythymidine monophosphate; dUMP: 2'deoxyuridine 5'-monophosphate (dUMP); FdUMP: 5-Fluoro-2'-deoxyuridine 5'-monophosphate (FdUMP); FnA: Folinic acid; GSH: Glutathione; HCC: Hepatocellular carcinoma; HMGB1: High mobility group protein B1; ICD: Immunogenic cell death; mAb: Monoclonal antibody; MDSCs: Myeloid derived suppressor cells (MDSCs); NAC: N-acetyl-L-cysteine; NP: Nanoparticle; OxP: Oxaliplatin; PEG: Polyethylene glycol; PFA: Paraformaldehyde; ROS: Reactive oxygen species; TEM: Transmission electron microscope; TME: Tumor microenvironment; Tregs: Regulatory T cells; 5-Fu: Fluorouracil

\section{Acknowledgements}

We thank Dr. Shulan Han for editorial assistance.

\section{Authors' contributions}

LH supervised the project. LH and JG designed the overall study, interpreted experiments, and wrote the paper. JG, DS, YZ performed physicochemical and in vitro experiments and analyzed data. JG, DS and YZ performed pharmacokinetics and in vivo toxicity experiments and analyzed data. JG, ZY and $Y L$ performed in vivo therapeutic experiments and analyzed data.

\section{Funding}

This study was supported by an NIH grant (CA198999) to LH. JG was supported by Health Commission of Jilin Province (2020Q012), Talents Cultivation Program of Jilin University, and Fundamental Research Funds for the Central Universities. ZY was supported by National Natural Science Foundation of China (81774240, 81403272), China Scholarship Counci (201808310047), Chenguang Program from Shanghai Education Development Foundation and Shanghai Municipal Education Commission (15CG48), Shanghai Rising-Star Program (17QA1403900), Training Plan of Outstanding Young Medical Talents from Shanghai Municipal Health Bureau (2017YQ021), and Talent Youth Supporting Project from Chinese Medicine Association (QNRC2-C14).

\section{Availability of data and materials}

All data generated during this study are included in this published article and its supplementary files.

\section{Ethics approval and consent to participate}

The procedures used in this study were approved by Institutional Animal Care and Use Committee of University of North Carolina at Chapel Hill and by the Animal Ethics Committee of Jilin University.

\section{Consent for publication}

Not applicable.

\section{Competing interests}

$\mathrm{LH}$ is a consultant of PDS Biotechnology, Samyang Biopharmaceutical Co, Stemirna and Beijing Inno Medicine. All other co-authors declare no conflict.

\section{Author details}

${ }^{1}$ School of Pharmaceutical Sciences, Jilin University, Changchun 130021 China. ${ }^{2}$ Division of Pharmacoengineering and Molecular Pharmaceutics, Eshelman School of Pharmacy, University of North Carolina, Chapel Hill, NC 27599, USA. ${ }^{3}$ Department of Hepatopathy, Shuguang Hospital, Affiliated to Shanghai University of Traditional Chinese Medicine, Shanghai 201203, China.
Received: 20 October 2020 Accepted: 17 December 2020

Published online: 06 January 2021

\section{References}

1. Allegra CJ, Yothers G, O'Connell MJ, Sharif S, Petrelli NJ, Lopa SH, Wolmark N. Bevacizumab in stage II-III colon cancer: 5-year update of the National Surgical Adjuvant Breast and bowel project C-08 trial. J Clin Oncol. 2013;31: 359-64.

2. Qin S, Bai Y, Lim HY, Thongprasert S, Chao Y, Fan J, Yang TS, Bhudhisawasdi $V$, Kang WK, Zhou Y, et al. Randomized, multicenter, open-label study of oxaliplatin plus fluorouracil/leucovorin versus doxorubicin as palliative chemotherapy in patients with advanced hepatocellular carcinoma from Asia. J Clin Oncol. 2013;31:3501-8.

3. Douillard JY, Siena S, Cassidy J, Tabernero J, Burkes R, Barugel M, Humblet Y, Bodoky G, Cunningham D, Jassem J, et al. Randomized, phase III trial of panitumumab with infusional fluorouracil, leucovorin, and oxaliplatin (FOLFOX4) versus FOLFOX4 alone as first-line treatment in patients with previously untreated metastatic colorectal cancer: the PRIME study. J Clin Oncol. 2010;28:4697-705.

4. Tournigand C, Cervantes A, Figer A, Lledo G, Flesch M, Buyse M, Mineur L, Carola E, Etienne PL, Rivera F, et al. OPTIMOX1: a randomized study of FOLFOX4 or FOLFOX7 with oxaliplatin in a stop-and-go fashion in advanced colorectal cancer--a GERCOR study. J Clin Oncol. 2006;24:394-400.

5. Andre T, Boni C, Mounedji-Boudiaf L, Navarro M, Tabernero J, Hickish T, Topham C, Zaninelli M, Clingan P, Bridgewater J, et al. Oxaliplatin, fluorouracil, and leucovorin as adjuvant treatment for colon cancer. N Engl J Med. 2004;350:2343-51.

6. Majumder J, Taratula O, Minko T. Nanocarrier-based systems for targeted and site specific therapeutic delivery. Adv Drug Deliv Rev. 2019;144:57-77.

7. Guo J, Yu Z, Das M, Huang L. Nano Codelivery of Oxaliplatin and Folinic acid achieves synergistic chemo-immunotherapy with 5-fluorouracil for colorectal Cancer and liver metastasis. ACS Nano. 2020;14:5075-89.

8. Guo J, Ogier JR, Desgranges S, Darcy R, O'Driscoll C. Anisamide-targeted cyclodextrin nanoparticles for siRNA delivery to prostate tumours in mice. Biomaterials. 2012;33:7775-84.

9. Shen L, Li J, Liu Q, Song W, Zhang X, Tiruthani K, Hu H, Das M, Goodwin TJ, Liu R, Huang L. Local blockade of interleukin 10 and C-X-C motif chemokine ligand 12 with Nano-delivery promotes antitumor response in murine cancers. ACS Nano. 2018;12:9830-41.

10. Song W, Shen L, Wang Y, Liu Q, Goodwin TJ, Li J, Dorosheva O, Liu T, Liu R, Huang L. Synergistic and low adverse effect cancer immunotherapy by immunogenic chemotherapy and locally expressed PD-L1 trap. Nat Commun. 2018:9:2237

11. Luan X, Rahme K, Cong Z, Wang L, Zou Y, He Y, Yang H, Holmes JD, O'Driscoll CM, Guo J. Anisamide-targeted PEGylated gold nanoparticles designed to target prostate cancer mediate: enhanced systemic exposure of siRNA, tumour growth suppression and a synergistic therapeutic response in combination with paclitaxel in mice. Eur J Pharm Biopharm. 2019;137:56-67

12. Tesniere A, Schlemmer F, Boige V, Kepp O, Martins I, Ghiringhelli F, Aymeric L, Michaud M, Apetoh L, Barault L, et al. Immunogenic death of colon cancer cells treated with oxaliplatin. Oncogene. 2010:29:482-91.

13. Lanzavecchia A, Sallusto F. Regulation of T cell immunity by dendritic cells. Cell. 2001:106:263-6.

14. Guermonprez P, Valladeau J, Zitvogel L, Thery C, Amigorena S. Antigen presentation and T cell stimulation by dendritic cells. Annu Rev Immunol. 2002;20:621-67.

15. Kroemer G, Galluzzi L, Kepp O, Zitvogel L. Immunogenic cell death in cancer therapy. Annu Rev Immunol. 2013;31:51-72

16. Duan X, Chan C, Han W, Guo N, Weichselbaum RR, Lin W. Immunostimulatory nanomedicines synergize with checkpoint blockade immunotherapy to eradicate colorectal tumors. Nat Commun. 2019;10:1899.

17. Chen Q, Chen J, Yang Z, Xu J, Xu L, Liang C, Han X, Liu Z. Nanoparticleenhanced radiotherapy to trigger robust Cancer immunotherapy. Adv Mater. 2019;31:e1802228.

18. Wang D, Wang T, Yu H, Feng B, Zhou L, Zhou F, Hou B, Zhang H, Luo M, Li $Y$. Engineering nanoparticles to locally activate $T$ cells in the tumor microenvironment. Sci Immunol. 2019;4:eaau6584.

19. Longley DB, Harkin DP, Johnston PG. 5-fluorouracil: mechanisms of action and clinical strategies. Nat Rev Cancer. 2003;3:330-8. 
20. Banerjee R, Tyagi P, Li S, Huang L. Anisamide-targeted stealth liposomes: a potent carrier for targeting doxorubicin to human prostate cancer cells. Int J Cancer. 2004;112:693-700.

21. Thapa RK, Margolis DJ, Kiick KL, O'Sullivan M. Enhanced wound healing via collagen-turnover-driven transfer of PDGF-BB gene in a murine wound model. ACS Appl Bio Mater. 2020;3:3500-17.

22. Zhang Y, Peng L, Mumper RJ, Huang L. Combinational delivery of c-myc siRNA and nucleoside analogs in a single, synthetic nanocarrier for targeted cancer therapy. Biomaterials. 2013;34:8459-68.

23. Guo S, Wang Y, Miao L, Xu Z, Lin CH, Huang L. Turning a water and oil insoluble cisplatin derivative into a nanoparticle formulation for cancer therapy. Biomaterials. 2014;35:7647-53.

24. Yu Z, Guo J, Hu M, Gao Y, Huang L. Icaritin exacerbates Mitophagy and synergizes with doxorubicin to induce immunogenic cell death in hepatocellular carcinoma. ACS Nano. 2020;14:4816-28.

25. Sun $H$, Yang W, Tian Y, Zeng X, Zhou J, Mok MTS, Tang W, Feng Y, Xu L, Chan AWH, et al. An inflammatory-CCRK circuitry drives mTORC1dependent metabolic and immunosuppressive reprogramming in obesityassociated hepatocellular carcinoma. Nat Commun. 2018;9:5214.

26. Wrightson WR, Myers SR, Galandiuk S. HPLC analysis of 5-FU and FdUMP in tissue and serum. Biochem Biophys Res Commun. 1995;216:808-13.

27. Song W, Tiruthani K, Wang Y, Shen L, Hu M, Dorosheva O, Qiu K, Kinghorn KA, Liu R, Huang L. Trapping of lipopolysaccharide to promote immunotherapy against colorectal Cancer and attenuate liver metastasis. Adv Mater. 2018;30:e1805007.

28. Hu M, Wang Y, Xu L, An S, Tang Y, Zhou X, Li J, Liu R, Huang L. Relaxin gene delivery mitigates liver metastasis and synergizes with check point therapy. Nat Commun. 2019;10:2993.

29. Das M, Shen L, Liu Q, Goodwin TJ, Huang L. Nanoparticle delivery of RIG-I agonist enables effective and safe adjuvant therapy in pancreatic Cancer. Mol Ther. 2019;27:507-17.

30. Goodwin TJ, Shen L, Hu M, Li J, Feng R, Dorosheva O, Liu R, Huang L. Liver specific gene immunotherapies resolve immune suppressive ectopic lymphoid structures of liver metastases and prolong survival. Biomaterials. 2017;141:260-71.

31. Hu K, Miao L, Goodwin TJ, Li J, Liu Q, Huang L. Quercetin remodels the tumor microenvironment to improve the permeation, retention, and antitumor effects of nanoparticles. ACS Nano. 2017;11:4916-25.

32. Cheng L, Wang Y, Huang L. Exosomes from M1-polarized macrophages potentiate the Cancer vaccine by creating a pro-inflammatory microenvironment in the lymph node. Mol Ther. 2017;25:1665-75.

33. Zhang $Y$, Schwerbrock NMJ, Rogers AB, Kim WY, Huang L. Codelivery of VEGF siRNA and gemcitabine monophosphate in a single nanoparticle formulation for effective treatment of NSCLC. Mol Ther. 2013;21:1559-69.

34. Yao J, Zhang Y, Ramishetti S, Wang YH, Huang L. Turning an antiviral into an anticancer drug: nanoparticle delivery of acyclovir monophosphate. J Control Release. 2013;170:414-20.

35. Li J, Chen YC, Tseng YC, Mozumdar S, Huang L. Biodegradable calcium phosphate nanoparticle with lipid coating for systemic siRNA delivery. J Control Release. 2010;142:416-21.

36. Li S, Wang A, Jiang W, Guan Z. Pharmacokinetic characteristics and anticancer effects of 5-fluorouracil loaded nanoparticles. BMC Cancer. 2008:8:103.

37. Safwat MA, Soliman GM, Sayed D, Attia MA. Fluorouracil-loaded gold nanoparticles for the treatment of skin Cancer: development, in vitro characterization, and in vivo evaluation in a mouse skin Cancer Xenograft model. Mol Pharm. 2018;15:2194-205.

38. Kazi J, Mukhopadhyay R, Sen R, Jha T, Ganguly S, Debnath MC. Design of 5fluorouracil (5-FU) loaded, folate conjugated peptide linked nanoparticles, a potential new drug carrier for selective targeting of tumor cells. Medchemcomm. 2019;10:559-72.

39. Saif MW, Syrigos KN, Katirtzoglou NA. S-1: a promising new oral fluoropyrimidine derivative. Expert Opin Investig Drugs. 2009;18:335-48.

40. Juliano RL. The delivery of therapeutic oligonucleotides. Nucleic Acids Res. 2016:44:6518-48.

41. Simon HU, Haj-Yehia A, Levi-Schaffer F. Role of reactive oxygen species (ROS) in apoptosis induction. Apoptosis. 2000:5:415-8.

42. Franco R, Cidlowski JA. Apoptosis and glutathione: beyond an antioxidant. Cell Death Differ. 2009;16:1303-14.

43. Lu SC. Regulation of glutathione synthesis. Mol Asp Med. 2009;30:42-59.
44. Chytil M, Pekar M. Effect of new hydrophobic modification of hyaluronan on its solution properties: evaluation of self-aggregation. Carbohydr Polym. 2009;76:443-339.

45. Bocci G, Danesi R, Di Paolo AD, Innocenti F, Allegrini G, Falcone A, Melosi A, Battistoni M, Barsanti G, Conte PF, Del Tacca M. Comparative pharmacokinetic analysis of 5-fluorouracil and its major metabolite 5-fluoro5,6-dihydrouracil after conventional and reduced test dose in cancer patients. Clin Cancer Res. 2000;6:3032-7.

46. Suk JS, Xu Q, Kim N, Hanes J, Ensign LM. PEGylation as a strategy for improving nanoparticle-based drug and gene delivery. Adv Drug Deliv Rev. 2016;99:28-51.

47. Wang YJ, Fletcher R, Yu J, Zhang L. Immunogenic effects of chemotherapyinduced tumor cell death. Genes Dis. 2018;5:194-203.

48. Showalter A, Limaye A, Oyer JL, Igarashi R, Kittipatarin C, Copik AJ, Khaled AR. Cytokines in immunogenic cell death: applications for cancer immunotherapy. Cytokine. 2017;97:123-32.

49. Hu M, Zhou X, Wang Y, Guan K, Huang L. Relaxin-FOLFOX-IL-12 triple combination therapy engages memory response and achieves long-term survival in colorectal cancer liver metastasis. J Control Release. 2020;319:213-21.

50. Litpins Q, Eppolito C, Odunsi K, Shrikant PA. IL-12-programmed long-term CD8+ T cell responses require STAT4. J Immunol. 2006;177:7618-25.

51. Raue HP, Beadling C, Haun J, Slifka MK. Cytokine-mediated programmed proliferation of virus-specific CD8(+) memory T cells. Immunity. 2013;38: 131-9.

52. Kasuya H, Kuruppu DK, Donahue JM, Choi EW, Kawasaki H, Tanabe KK. Mouse models of subcutaneous spleen reservoir for multiple portal venous injections to treat liver malignancies. Cancer Res. 2005;65:3823-7.

53. Guo J, Huang L. Membrane-core nanoparticles for cancer nanomedicine. Adv Drug Deliv Rev. 2020. https://doi.org/10.1016/j.addr.2020.05.005.

54. van der Meel R, Sulheim E, Shi Y, Kiessling F, Mulder WJM, Lammers T. Smart cancer nanomedicine. Nat Nanotechnol. 2019;14:1007-17.

55. Roxburgh CS, Shia J, Vakiani E, Daniel T, Weiser MR. Potential immune priming of the tumor microenvironment with FOLFOX chemotherapy in locally advanced rectal cancer. Oncoimmunology. 2018;7:e1435227.

56. Guan Y, Kraus SG, Quaney MJ, Daniels MA, Mitchem JB, Teixeiro E. FOLFOX chemotherapy ameliorates CD8 T lymphocyte exhaustion and enhances checkpoint blockade efficacy in colorectal Cancer. Front Oncol. 2020;10:586.

57. Sinicrope FA, Sargent DJ. Molecular pathways: microsatellite instability in colorectal cancer: prognostic, predictive, and therapeutic implications. Clin Cancer Res. 2012;18:1506-12.

58. Ruiz-Banobre J, Goel A. DNA mismatch repair deficiency and immune checkpoint inhibitors in gastrointestinal cancers. Gastroenterology. 2019;156: 890-903.

59. Liu Y, Guo J, Huang L. Modulation of tumor microenvironment for immunotherapy: focus on nanomaterial-based strategies. Theranostics. 2020; 10:3099-117.

60. Yang Y, Guo J, Huang L. Tackling TAMs for Cancer immunotherapy: It's Nano time. Trends Pharmacol Sci. 2020;41:701-14.

61. Galon J, Bruni D. Approaches to treat immune hot, altered and cold tumours with combination immunotherapies. Nat Rev Drug Discov. 2019;18:197-218.

62. Castle JC, Loewer M, Boegel S, de Graaf J, Bender C, Tadmor AD, Boisguerin $V$, Bukur T, Sorn P, Paret $C$, et al. Immunomic, genomic and transcriptomic characterization of CT26 colorectal carcinoma. BMC Genomics. 2014;15(190).

63. Germano G, Lamba S, Rospo G, Barault L, Magri A, Maione F, Russo M, Crisafulli G, Bartolini A, Lerda G, et al. Inactivation of DNA repair triggers neoantigen generation and impairs tumour growth. Nature. 2017;552:116-20.

64. Bjornmalm M, Thurecht KJ, Michael M, Scott AM, Caruso F. Bridging bioNano science and Cancer Nanomedicine. ACS Nano. 2017;11:9594-613.

65. van der Meel R, Lammers T, Hennink WE. Cancer nanomedicines: oversold or underappreciated? Expert Opin Drug Deliv. 2017;14:1-5.

66. Gustavsson B, Carlsson G, Machover D, Petrelli N, Roth A, Schmoll HJ, Tveit KM, Gibson F. A review of the evolution of systemic chemotherapy in the management of colorectal cancer. Clin Colorectal Cancer. 2015;14:1-10.

\section{Publisher's Note}

Springer Nature remains neutral with regard to jurisdictional claims in published maps and institutional affiliations. 